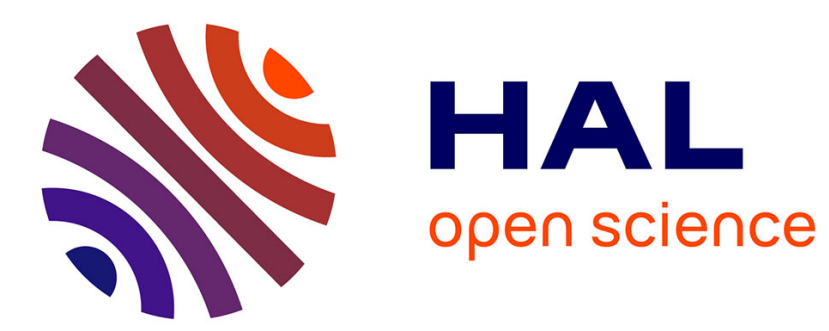

\title{
Wall laws for fluid flows at a boundary with random roughness
}

\author{
Arnaud Basson, David Gerard-Varet
}

\section{To cite this version:}

Arnaud Basson, David Gerard-Varet. Wall laws for fluid flows at a boundary with random roughness. 2006. hal-00083222

\section{HAL Id: hal-00083222 \\ https://hal.science/hal-00083222}

Preprint submitted on 29 Jun 2006

HAL is a multi-disciplinary open access archive for the deposit and dissemination of scientific research documents, whether they are published or not. The documents may come from teaching and research institutions in France or abroad, or from public or private research centers.
L'archive ouverte pluridisciplinaire HAL, est destinée au dépôt et à la diffusion de documents scientifiques de niveau recherche, publiés ou non, émanant des établissements d'enseignement et de recherche français ou étrangers, des laboratoires publics ou privés. 


\title{
Wall laws for fluid flows at a boundary with random roughness
}

\author{
*Arnaud Basson, ${ }^{\dagger}$ David GÉRARD-VARET
}

June 29, 2006

The general concern of this paper is the effect of rough boundaries on fluids. We consider a stationary flow, governed by incompressible Navier-Stokes equations, in an infinite domain bounded by two horizontal rough plates. The roughness is modeled by a spatially homogeneous random field, with characteristic size $\varepsilon$. A mathematical analysis of the flow for small $\varepsilon$ is performed. The Navier's wall law is rigorously deduced from this analysis. This extends substantially former results obtained in the case of periodic roughness, notably in [15, 16].

\section{Contents}

\begin{tabular}{lll}
\hline 1 & Introduction & 2
\end{tabular}

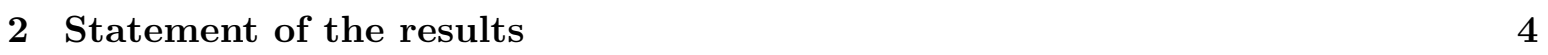

2.1 Modeling of the rough domain . . . . . . . . . . . . . . . . . 4

2.2 Main results . . . . . . . . . . . . . . . . . . . . . . 6

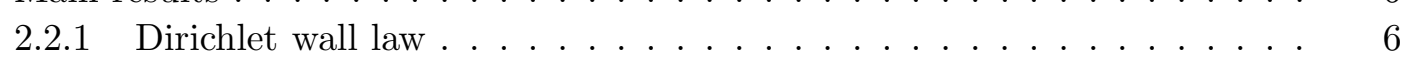

2.2 .2 Navier's wall law $\ldots \ldots \ldots \ldots \ldots$

$\begin{array}{lll}3 & \text { Justification of Dirichlet wall law } & 10\end{array}$

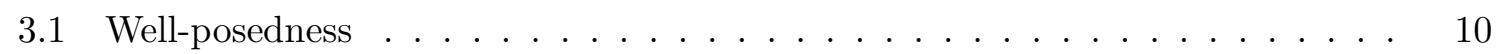

$3.1 .1 \quad$ Linear problem . . . . . . . . . . . . . . . . . . . . . . 11

$3.1 .2 \quad$ Nonlinear problem . . . . . . . . . . . . . . . . . . . . . 15

$3.1 .3 \quad$ Measurability . . . . . . . . . . . . . . . . . . . . 15

3.2 Estimates for Dirichlet wall law . . . . . . . . . . . . . . . . . 17

3.2 .1 Two lemmas for the Stokes system . . . . . . . . . . . . . . . . 18

3.2 .2 The duality estimate $\ldots \ldots \ldots \ldots \ldots \ldots \ldots$. . . . . . . . . . . . . 20

\begin{tabular}{|lll}
\hline 4 & The boundary layer analysis & 23
\end{tabular}

4.1 Formal expansion . . . . . . . . . . . . . . . . . . . . . . . . 23

4.2 Well-posedness of $(2.7) \ldots \ldots \ldots \ldots \ldots \ldots \ldots$

$4.2 .1 \quad$ Stochastic derivative, Convolution . . . . . . . . . . . . . 24

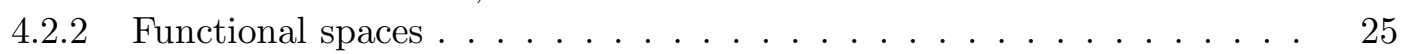

4.2 .3 Variational formulation . . . . . . . . . . . . . . . . 26

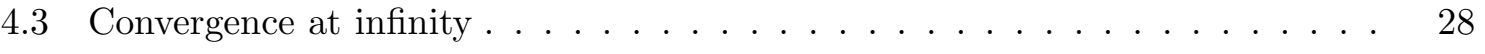

*DMA, Ecole Normale Supérieure, 45 rue d'Ulm,75005 Paris

${ }^{\dagger}$ DMA/CNRS, Ecole Normale Supérieure, 45 rue d'Ulm,75005 Paris 


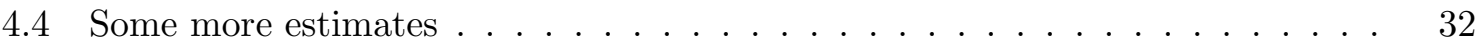

\begin{tabular}{|lll}
5 & Justification of Navier's law & 33
\end{tabular}

5.1 Approximation of $u^{\varepsilon} \ldots \ldots \ldots \ldots \ldots \ldots \ldots$

$5.1 .1 \quad$ Construction of $v_{l}$ and $v_{u} \ldots \ldots \ldots \ldots \ldots \ldots$

5.1 .2 Proof of theorem $4 \ldots \ldots \ldots \ldots \ldots$

5.2 Effective wall law $\ldots \ldots \ldots \ldots \ldots \ldots \ldots \ldots \ldots$

\section{Introduction}

The understanding of roughness-induced effects is a major concern in fluid dynamics. Indeed, many examples of physical relevance involve rough boundaries. By "rough", we mean that the spatial variations are small compared to the typical length of the problem. For instance, in geophysics, the bottom of the oceans and the shores are rough with respect to the large scale flow. Also, in an industrial framework, containers have often imperfections that qualify them as rough.

The main problem is to know in which way such irregular boundaries affect the flow. This is especially important with regards to numerical computations: indeed, roughness is in general too small to be captured by the discretization grid of the simulations.

To overcome this difficulty, one often relies on wall laws. A wall law is a boundary condition that is imposed on an artificial boundary inside the domain. The idea is to filter out the precise description of the flow near the real rough boundary. The wall law should only reflect the large scale effect of the roughness, in the spirit of a homogeneization process.

In many cases, the determination of wall laws relies on formal calculations, grounded by empirical arguments (see for instance [6, 20]). The present paper is a mathematical justification of some wall laws, in the case of an incompressible viscous fluid. We consider the two-dimensional stationary Navier-Stokes equations:

$$
\left\{\begin{array}{l}
u \cdot \nabla u+\nabla p-\nu \Delta u=0, x \in \Omega^{\varepsilon}, \\
\operatorname{div} u=0, x \in \Omega^{\varepsilon},
\end{array}\right.
$$

in a domain $\Omega^{\varepsilon}$ of channel type:

$$
\Omega^{\varepsilon}=\left\{\left(x_{1}, x_{2}\right) \in \mathbb{R}^{2}, \gamma_{l}^{\varepsilon}\left(x_{1}\right)<x_{2}<\gamma_{u}^{\varepsilon}\left(x_{1}\right)\right\},
$$

where the lower and upper boundaries $\gamma_{l}^{\varepsilon}$ and $\gamma_{u}^{\varepsilon}$ are to be precised. As usual, the fields $u=\left(u_{1}, u_{2}\right)(x) \in \mathbb{R}^{2}, \quad p=p(x) \in \mathbb{R}$ are the velocity and the pressure, and $\nu>0$ is the kinematic viscosity. Equations (1.1) are completed with the classical no-slip conditions

$$
\left.u\right|_{\partial \Omega^{\varepsilon}}=0 .
$$

Moreover, we prescribe the fluid flux through the channel, that is condition

$$
\int_{\sigma\left(x_{1}\right)} u_{1}=\phi
$$

where $\phi>0$ is a given constant flux, and

$$
\sigma\left(x_{1}\right)=\left\{\gamma_{l}^{\varepsilon}\left(x_{1}\right)<x_{2}<\gamma_{u}^{\varepsilon}\left(x_{1}\right)\right\}
$$


is a vertical section of the channel at $x_{1}$. Remark that by incompressibility and boundary condition (1.2), the left hand-side of (1.3) does not depend on $x_{1}$. The functions $\gamma_{l}^{\varepsilon}$ and $\gamma_{u}^{\varepsilon}$ model rough plates, with small characteristic size $\varepsilon$. Broadly, they read:

$$
\gamma_{l}^{\varepsilon}=-\varepsilon \gamma_{l}\left(x_{1} / \varepsilon\right), \quad \gamma_{u}^{\varepsilon}=1+\varepsilon \gamma_{u}\left(x_{1} / \varepsilon\right),
$$

for Lipschitz functions

$$
\left.\gamma_{l}=\gamma_{l}\left(y_{1}\right) \in\right] 0,1\left[, \quad \gamma_{u}=\gamma_{u}\left(y_{1}\right) \in\right] 0,1[.
$$

More precise assumptions on $\gamma_{l}$ and $\gamma_{u}$ will be made further on. The set $\left.\Omega=\mathbb{R} \times\right] 0,1[$ will be called the interior domain.

We wish to study solutions $u^{\varepsilon}$ of (1.1), (1.2), (1.3), and to determine appropriate wall laws for this system. In other words, we look for operators $\mathcal{B}^{\varepsilon}\left(x, D_{x}\right)$ such that solutions $v^{\varepsilon}$ of the interior system

$$
\left\{\begin{array}{l}
v \cdot \nabla v+\nabla q-\nu \Delta v=0, x \in \Omega, \\
\operatorname{div} v=0, x \in \Omega, \\
\int_{\sigma\left(x_{1}\right)} v_{1}=\phi, \\
\left.\mathcal{B}^{\varepsilon}\left(x, D_{x}\right)(v)\right|_{\partial \Omega}=0,
\end{array}\right.
$$

approximate well $u^{\varepsilon}$ in $\Omega$, for small $\varepsilon$.

The mathematical treatment of wall laws has been the matter of many articles. The note [4] is devoted to the analysis of Laplace equation in an annular domain with perforations. Numerical and formal computations for fluid flows can be found in [2] [3]. An analysis of Couette flows in rough domains has been performed in [5]. Let us also mention the important contributions of Jäger and Mikelic on wall laws for channel flows ([15, 16]). We refer to [17] on a related problem with porous boundaries. Finally, see [14], [9] for study of roughness-induced effects on some geophysical systems.

All these articles are devoted to periodic roughness, meaning that the boundary functions $\gamma_{l}$ and $\gamma_{u}$ are periodic. This is of course a mathematical simplification, which is highly unrealistic from the point of view of physics. Our goal here is to drop this restriction, and treat non-periodic roughness. Precisely, we consider roughness that is distributed following a spatially homogeneous random field. A complete description of the rough domain will be given in the next section.

Following [15] in the periodic case, special attention is paid to the simple Dirichlet wall law:

$$
\left.\mathcal{B}^{\varepsilon}\left(x, D_{x}\right)(v)\right|_{\partial \Omega}=\left.v\right|_{\partial \Omega}=0,
$$

and to the Navier's friction law:

$$
\left.\mathcal{B}^{\varepsilon}\left(x, D_{x}\right)(v)\right|_{\partial \Omega}=\left.\left(C_{\varepsilon}(x) \frac{\partial v_{\tau}}{\partial n}-v_{\tau}\right)\right|_{\partial \Omega}=0 .
$$

introduced by Navier [21] and extensively used in simulations of geophysical flows. Losely, we show two main results:

1. The Dirichlet wall law yields a $O(\varepsilon)$ approximation of the real solution $u^{\varepsilon}$, that is $u^{\varepsilon}-v^{\varepsilon}$ is $O(\varepsilon)$ in an appropriate quadratic norm, to be described in the next section. 
2. For appropriate $C_{\varepsilon}$, the Navier's law yields a $o(\varepsilon)$ approximation of the real solution $u^{\varepsilon}$.

These results extend those of [15]. They are deduced for a precise description of $u^{\varepsilon}$ for small $\varepsilon$, especially of the boundary layer flow near $\partial \Omega^{\varepsilon}$. Precise statements, including the expression of $C_{\varepsilon}$, will be given in the next section.

To end this introduction, let us point out some difficulties related to the proof of these results. First, we consider a domain $\Omega^{\varepsilon}$ that is not bounded in the tangential direction $\left(x_{1} \in \mathbb{R}\right)$. To our knowledge, previous studies dealt with bounded channel domains, wether with lateral boundaries (plus in- and out-flux lateral boundary conditions, see [15]) or with periodic boundary conditions, see [5]. Note that such periodicity condition is not compatible with our non-periodic roughness. Due to the unbounded channel domain, we work with only locally integrable functions, which leads to completely different treatment of the energy estimates. Secondly, as the roughness is non-periodic, the boundary layer system is more complex. Due to the lack of compactness both in the tangential and transverse variables, we are not able to solve it in a deterministic setting. We use a variational formulation that involves the random variable. In addition to this problem, the behaviour of the boundary layer profile far from the boundary is not obvious. This can be understood using formally the tangential Fourier transform. Indeed, in the periodic setting, the Fourier modes are discrete, and allow a clear separation between the non-oscillating part (the constant mode) and the oscillating ones (the non-constant modes). But in the non-periodic case, there is no such separation, and Fourier modes close to zero create trouble. To control these low frequencies, we must again inject some probabilistic information (namely, the ergodic theorem). This difficulty appears also further on in the study, to establish energy estimates.

The rest of the paper is structured as follows. The next section contains a precise modeling of the domain, and the statements of the mathematical results. The third section is devoted to the Dirichlet wall law. The fourth section focuses on the boundary layer analysis. The final section is the justification of Navier's wall law.

\section{Statement of the results}

\subsection{Modeling of the rough domain}

Let $\varepsilon>0$, and $(M, \mathcal{M}, \mu)$ a probability space. For all $m \in M$, we define a rough domain $\Omega^{\varepsilon}(m)$ by

$$
\Omega^{\varepsilon}(m)=\Omega \cup R_{l}^{\varepsilon}(m) \cup R_{u}^{\varepsilon}(m),
$$

where $\Omega=\mathbb{R} \times] 0,1\left[\right.$ is the interior domain, and $R_{l . u}^{\varepsilon}(m)$ is the lower, upper rough part. To obtain a realistic model for roughness, we use spatially homogeneous random fields: following [22], [18] or [8], we recall that a homogeneous random field is a measurable map

$$
\gamma: M \times \mathbb{R}^{n} \mapsto \mathbb{R}^{m}
$$

satisfying: for all $h, z_{1}, \ldots, z_{k}$ and all Borel subsets $B_{1}, \ldots, B_{k}$ of $\mathbb{R}^{m}$,

$$
\begin{aligned}
\mu\left(\left\{m \in M, \gamma\left(m, z_{1}+h\right) \in B_{1}, \ldots, \gamma\left(m, z_{k}+h\right) \in B_{k}\right\}\right) & \\
& =\mu\left(\left\{m \in M, \gamma\left(m, z_{1}\right) \in B_{1}, \ldots, \gamma\left(m, z_{k}\right) \in B_{k}\right\}\right) .
\end{aligned}
$$


We remind that for $n=m=1$, a homogeneous random field is often called a stationary random process. We thus define

$$
\begin{aligned}
& R_{l}^{\varepsilon}(m)=\left\{x=\left(x_{1}, x_{2}\right), x_{1} \in \mathbb{R}^{2}, 0>x_{2}>-\varepsilon \gamma_{l}\left(m, x_{1} / \varepsilon\right)\right\}, \\
& R_{u}^{\varepsilon}(m)=\left\{x=\left(x_{1}, x_{2}\right), x_{1} \in \mathbb{R}^{2}, 0<x_{2}-1<\varepsilon \gamma_{u}\left(m, x_{1} / \varepsilon\right)\right\},
\end{aligned}
$$

where $\left.\left(\gamma_{l}, \gamma_{u}\right)=\left(\gamma_{l}, \gamma_{u}\right)\left(m, y_{1}\right) \in\right] 0,1\left[^{2}\right.$ is a homogeneous random field. Moreover, we assume that for all $m, \gamma_{l, u}(m, \cdot)$ is a K-Lipschitz function, with $K>0$ independent of $m$, and that $m \mapsto \gamma_{l, u}(m, \cdot)$ is measurable with values in the set $C_{b}\left(\mathbb{R} ; \mathbb{R}^{2}\right)$ of continuous bounded functions.

Following a classical construction of Doob (see [12] or [8] for all necessary details), we introduce another probability space, which will be more convenient to our description. Let $P$ the set of K-Lipschitz functions $\omega: \mathbb{R} \mapsto] 0,1\left[{ }^{2}\right.$. Let $\mathcal{P}$ the $\sigma$-algebra generated by the sets

$$
\left\{\omega \in P, \omega\left(y_{1}\right) \in A, \forall y_{1} \in B\right\},
$$

where $B$ is a finite subset of $\mathbb{Q}$, and $A$ is a disk with rational center and radius. Note that $\mathcal{P}$ is simply the borelian $\sigma$-algebra of $P$, seen as a subset of $C_{b}\left(\mathbb{R} ; \mathbb{R}^{2}\right)$. Finally, consider the set function $\pi: \mathcal{P} \mapsto \mathbb{R}$ given by

$$
\pi(Q)=\mu\left(\left\{m \in M,\left(\gamma_{u}, \gamma_{l}\right)(m, \cdot) \in Q\right\}\right) .
$$

One can show $(c f[12])$ that $\pi$ is a probability measure on $(P, \mathcal{P})$. Moreover, we can define a translation group

$$
\tau_{h}: P \mapsto P, \quad \tau_{h}(\omega)\left(y_{1}\right)=\omega\left(y_{1}+h\right), \quad \forall y_{1}, h \in \mathbb{R},
$$

that preserves $\pi$. In this way, one can also describe the boundaries with the measurable map

$$
\left(\omega_{l}, \omega_{u}\right)\left(y_{1}, \omega\right)=\omega\left(y_{1}\right)
$$

Indeed, the laws of the random variables

$$
\left(\left(\gamma_{l}, \gamma_{u}\right)\left(\cdot, z_{1}+h\right), \ldots,\left(\gamma_{l}, \gamma_{u}\right)\left(\cdot, z_{k}+h\right)\right)
$$

and

$$
\left(\left(\omega_{l}, \omega_{u}\right)\left(\cdot, z_{1}+h\right), \ldots,\left(\omega_{l}, \omega_{u}\right)\left(\cdot, z_{k}+h\right)\right)
$$

are the same (and independent of $h$, for any $z_{1}, \ldots, z_{k}$ ). The advantage of this last framework is that one can write

$$
\omega_{u, l}\left(y_{1}, \omega\right)=h_{u, l} \circ \tau_{y_{1}}(\omega), \text { with }\left(h_{u}, h_{l}\right)(\omega)=\omega(0),
$$

which will be useful in the study of boundary layers. Hence, we will rather use the formulation in terms of $\omega, h_{u}, h_{l}$, and consider, for all $\omega \in P$,

$$
\Omega^{\varepsilon}(\omega),=\Omega \cup R_{l}^{\varepsilon}(\omega) \cup R_{u}^{\varepsilon}(\omega),
$$

where $\Omega=\mathbb{R} \times] 0,1[$,

$$
\begin{aligned}
& R_{l}^{\varepsilon}(\omega)=\left\{x=\left(x_{1}, x_{2}\right), 0>x_{2}>-\varepsilon h_{l} \circ \tau_{x_{1} / \varepsilon}(\omega)\right\}, \\
& R_{u}^{\varepsilon}(\omega)=\left\{x=\left(x_{1}, x_{2}\right), 0<x_{2}-1<\varepsilon h_{u} \circ \tau_{x_{1} / \varepsilon}(\omega)\right\} .
\end{aligned}
$$




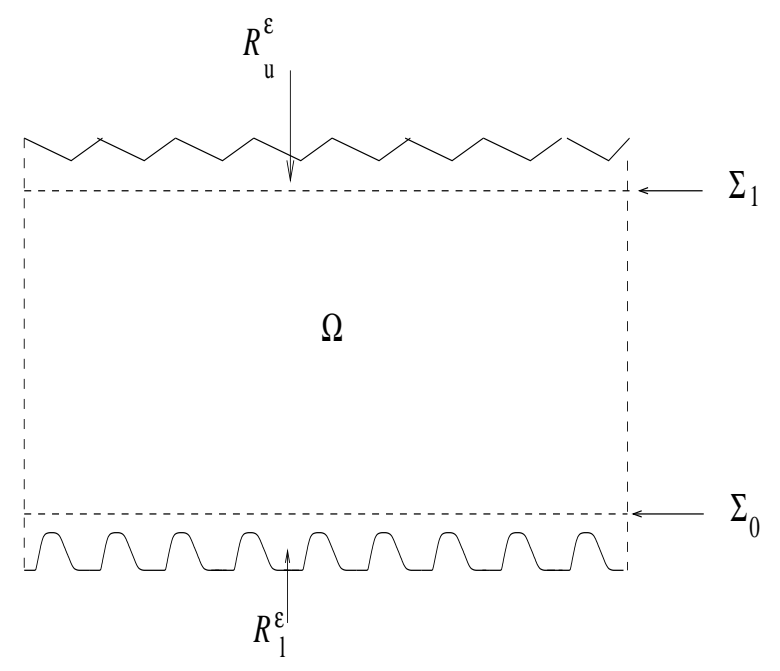

Figure 1: The rough domain $\Omega^{\varepsilon}$.

We also define $\Sigma_{0}=\mathbb{R} \times\{0\}, \Sigma_{1}=\mathbb{R} \times\{1\}$ the horizontal boundaries of $\Omega$.

Note that our modeling is derived from stochastic homogeneization, where domains with small holes are described with such random fields (see again [22], [18], [11], [1], [7] among others). However, we emphasize that the classical tools of homogeneization (such as compensated compactness, two-scale convergence) do not apply to our boundary problem: broadly, boundary layers are not seen in weak convergence processes, so that they do not allow to recover precise energy estimates. To prove the theorems of the next sections will require a precise construction of approximate solutions.

Note also that this random framework includes the periodic one. Take $P=\mathbb{T}$ the unit torus, $\mathcal{P}$ its borelian $\sigma$-algebra, and $\pi$ the Lebesgue measure. For any periodic function $F$ on $\mathbb{T}$, any $y_{1}, \omega$ in $\mathbb{T}$ the formula

$$
f\left(y_{1}, \omega\right)=F\left(y_{1}+\omega\right)=F\left(\tau_{y_{1}}(\omega)\right)
$$

allows to "randomize" the periodic structure.

In addition to the rough domain $\Omega^{\varepsilon}$, we need to define boundary layer domains, that will be useful in the study of Navier's wall law. Namely,

$$
\begin{aligned}
& \mathcal{R}_{l}(\omega)=\left\{y=\left(y_{1}, y_{2}\right), y_{2}>h_{l} \circ \tau_{y_{1}}(\omega)\right\}, \\
& \mathcal{R}_{u}(\omega)=\left\{y=\left(y_{1}, y_{2}\right), y_{2}<h_{u} \circ \tau_{y_{1}}(\omega)\right\} .
\end{aligned}
$$

We will denote

$$
\mathcal{R}_{l, u}(\omega)=\mathcal{R}_{l, u}^{+}(\omega) \cup \Sigma_{0} \cup \mathcal{R}_{l, u}^{-}(\omega),
$$

with

$$
\mathcal{R}_{l, u}^{ \pm}(\omega)=\mathcal{R}_{l, u}(\omega) \cap\left\{ \pm y_{2}>0\right\}
$$

\subsection{Main results}

\subsubsection{Dirichlet wall law}

The first step in the analysis of system (1.1), (1.2), (1.3) is to prove the existence and uniqueness of solutions. For given parameters $\varepsilon, \omega$, this question has been adressed by Ladyženskaja 
and Solonnikov in article [19]. We also refer to [13] for good overview on channel flow problems. The results of [19] yield existence and uniqueness of a solution $u^{\varepsilon}(\omega, \cdot)$ in the space

$$
B_{2}\left(\Omega^{\varepsilon}(\omega)\right)=\left\{u \in H_{0, l o c}^{1}\left(\Omega^{\varepsilon}(\omega)\right), \quad \sup _{R \geq 1} \frac{1}{R} \int_{\Omega^{\varepsilon}(\omega, R)}|\nabla u|^{2}<+\infty\right\},
$$

where

$$
\Omega^{\varepsilon}(\omega, R)=\Omega^{\varepsilon}(\omega) \cap\left\{0<\left|x_{1}\right|<R\right\}, \quad R>0,
$$

for small enough flux $\phi<\phi_{0}$. However, we can not apply this result, as the dependence of $\phi_{0}$ with respect to $\varepsilon$ and $\omega$, as well as measurability properties with respect to $\omega$ are not clear. Hence, we show directly the following existence and uniqueness result:

Theorem 1 There exists $\phi_{0}>0$ and $\varepsilon_{0}>0$ such that: for all $\phi<\phi_{0}$, for all $\varepsilon<\varepsilon_{0}$, for all $\omega \in P$, system (1.1), (1.2), (1.3) has a unique solution

$$
u^{\varepsilon}(\omega, \cdot) \in B_{2}\left(\Omega^{\varepsilon}(\omega)\right) .
$$

Moreover, if we denote $\tilde{u}^{\varepsilon}(\omega, \cdot)$ the zero-extension of $u^{\varepsilon}(\omega, \cdot)$ outside $\Omega^{\varepsilon}(\omega)$, then the mapping

$$
P \mapsto H_{l o c}^{1}\left(\mathbb{R}^{2}\right), \quad \omega \mapsto \tilde{u}^{\varepsilon}(\omega, \cdot)
$$

is measurable.

As will be clear from the proof, the solution $u^{\varepsilon}$ is a perturbation of the following flow:

$$
\left\{\begin{array}{l}
u^{0}(\omega, x)=\left(6 \phi x_{2}\left(1-x_{2}\right), 0\right), x \in \Omega \\
u^{0}(\omega, x)=0, x \in \Omega^{\varepsilon}-\Omega .
\end{array}\right.
$$

Note that in restriction to $\Omega, u^{0}$ is simply the Poiseuille flow, that is the solution of (1.4), (1.5). We show the following estimates

Theorem 2 There exists $C>0$ such that, for all $\omega \in P$ :

$$
\left\{\begin{array}{l}
\sup _{R \geq 1} \frac{1}{R} \int_{\Omega^{\varepsilon}(\omega, R)}\left|\nabla u^{\varepsilon}(\omega, \cdot)-\nabla u^{0}(\omega, \cdot)\right|^{2} \leq C \varepsilon \\
\sup _{R \geq 1} \frac{1}{R} \int_{\Sigma_{0}(R) \cup \Sigma_{1}(R)}\left|u^{\varepsilon}(\omega, \cdot)\right|^{2} \leq C \varepsilon^{2} \\
\sup _{R \geq 1} \frac{1}{R} \int_{\Omega(R)}\left|u^{\varepsilon}(\omega, \cdot)-u^{0}(\omega, \cdot)\right|^{2} \leq C \varepsilon^{2}
\end{array}\right.
$$

where

$$
\Omega(R)=\Omega \cap\left\{0<\left|x_{1}\right|<R\right\}, \quad \Sigma_{0,1}(R)=\Sigma_{0,1} \cap\left\{0<\left|x_{1}\right|<R\right\} .
$$

Theorem 2.5 expresses that the Dirichlet wall law yields a $O(\varepsilon)$ quadratic approximation of the real solution. The obtention of the $L^{2}$-estimate relies on a duality argument. 


\subsubsection{Navier's wall law}

The Dirichlet wall law, that is the approximation of $u^{\varepsilon}$ by $u^{0}$ does not account for the behaviour of $u^{\varepsilon}$ near the rough bondaries. To derive a more accurate wall law, we carry a boundary layer analysis. We show that, for small $\varepsilon, u^{\varepsilon}$ is close to

$$
\begin{aligned}
u_{a p p}^{\varepsilon}(\omega, x)= & u^{0}(\omega, x)+\varepsilon U_{l}\left(\omega, \frac{x_{1}}{\varepsilon}, \frac{x_{2}}{\varepsilon}\right)+\varepsilon U_{u}\left(\omega, \frac{x_{1}}{\varepsilon}, \frac{x_{2}-1}{\varepsilon}\right) \\
& +\varepsilon u^{1}(\omega, x),
\end{aligned}
$$

where $U_{u, l}$ is an appropriate boundary layer term, and $u^{1}$ an appropriate Poiseuille type flow. Precisely, $U_{l, u}=U_{l . u}(\omega, y)$ satisfies a Stokes system with jump conditions:

$$
\left\{\begin{array}{l}
-\nu \Delta U_{l, u}(\omega, \cdot)+\nabla P_{l, u}(\omega, \cdot)=0, \quad y \in \mathcal{R}_{l, u}^{ \pm}(\omega) \\
\operatorname{div} U_{l, u}(\omega, \cdot)=0, \quad y \in \mathcal{R}_{l, u}(\omega), \\
{\left.\left[U_{l, u}(\omega, \cdot)\right]\right|_{\Sigma_{0}}=0,} \\
{\left.\left[\partial_{2} U_{l, u}(\omega, \cdot)-P_{l, u}(\omega, \cdot) e_{2}\right]\right|_{\Sigma_{0}}=-6 \phi} \\
U_{l, u}=0, \quad y \in \partial \mathcal{R}_{l, u}(\omega) .
\end{array}\right.
$$

In the case of periodic roughness, the solvability of system (2.7) is direct. Morever, using the Fourier transform in tangential variables, it can be seen easily that $U_{l, u}$ converges exponentially fast to a constant as $y_{2}$ goes to infinity. The random setting requires much more work. We prove in section 1 the following

Theorem 3 For all $\phi>0$, there exits a unique variational solution $U_{l, u}$ of (2.7), in the sense of paragraph 4.2.9. It is almost surely a classical solution, and satisfies

$$
\begin{aligned}
& \sup _{R \geq 1} \frac{1}{R} \mathbb{E}\left(\int_{\mathcal{R}_{l, u}(\cdot, R)}\left|\nabla U_{l, u}(\cdot, y)\right|^{2} d y\right)<+\infty, \\
& \sup _{R \geq 1} \frac{1}{R} \int_{\mathcal{R}_{l, u}(\omega, R)}\left|\nabla U_{l, u}(\omega, y)\right|^{2} d y<\infty \quad \text { almost surely, }
\end{aligned}
$$

where as usual

$$
\mathcal{R}_{l, u}(\omega, R)=\mathcal{R}_{l, u}(\omega) \cap\left\{0<\left|y_{1}\right|<R\right\} .
$$

Moreover, there exists a measurable map: $U_{l, u}^{\infty}: P \mapsto \mathbb{R}^{2}$, with $U_{l, u, 2}^{\infty}=0$, such that

$$
\begin{aligned}
& \sup _{R \geq 1} \mathbb{E}\left(\frac{1}{R} \int_{\left|y_{1}\right|<R}\left|U_{l, u}\left(\omega, y_{1}, y_{2}\right) d y_{1}-U_{l, u}^{\infty}(\omega)\right|^{2} d y_{1}\right) \underset{y_{2} \rightarrow \infty}{\longrightarrow} 0 \\
& \left|U_{l, u}\left(\omega, y_{1}, y_{2}\right)-U_{l, u}^{\infty}(\omega)\right| \underset{y_{2} \rightarrow \infty}{\longrightarrow} 0, \text { locally uniformly in } y_{1} .
\end{aligned}
$$

The convergence of $U_{l, u}$ towards a constant is proved using the ergodic theorem. Contrary to the periodic case, we are not able to precise the speed of convergence (see section $\$$ for all details).

We then establish the estimates on $u^{\varepsilon}-u_{a p p}^{\varepsilon}$ : 
Theorem 4 Let $U_{l, u}$ as in theorem 3, $u^{1}$ given in (4.2). The approximation $u_{\text {app }}^{\varepsilon}$ of (2.6) satisfies, as $\varepsilon \rightarrow 0$ :

$$
\left\{\begin{array}{l}
\sup _{R \geq 1} \frac{1}{R} \mathbb{E}\left(\int_{\Omega^{\varepsilon}(\cdot, R)}\left|\nabla u^{\varepsilon}(\omega, \cdot)-\nabla u_{\text {app }}^{\varepsilon}\right|^{2}\right)=o\left(\varepsilon^{2}\right), \\
\sup _{R \geq 1} \frac{1}{R} \mathbb{E}\left(\int_{\Omega(R)}\left|u^{\varepsilon}(\omega, \cdot)-u_{\text {app }}^{\varepsilon}(\omega, \cdot)\right|^{2}\right)=o\left(\varepsilon^{2}\right),
\end{array}\right.
$$

In the periodic setting, for which the boundary layer profiles $U_{l, u}-U_{l, u}^{\infty}$ decay exponentially with $y_{2}$, the bound $o\left(\varepsilon^{2}\right)$ turns to $O\left(\varepsilon^{3}\right)$ for the $H^{1}$ estimate, and $O\left(\varepsilon^{4}\right)$ for the $L^{2}$ estimate. But in the general random setting, we are not able to improve the bound $o\left(\varepsilon^{2}\right)$ : it is related to the speed of convergence of $U_{l, u}$ as $y_{2} \rightarrow+\infty$. Theorem 1 extends the results of Jäger and Mikelic, [15, theorem 1, p113] for periodic roughness. As they consider domains with lateral boundaries, they need to add a lateral boundary layer term, which yields a less precise estimate: the $O\left(\varepsilon^{3}\right)$ and $O\left(\varepsilon^{4}\right)$ bounds are replaced by $O\left(\varepsilon^{2}\right)$ and $O\left(\varepsilon^{3}\right)$ bounds respectively. The unbounded setting allows to avoid this loss of accuracy.

We may now justify the relevance of Navier's friction law. Let $v^{\varepsilon}(\omega, \cdot)$ the solution of

$$
\left\{\begin{array}{l}
v \cdot \nabla v+\nabla q-\nu \Delta v=0, x \in \Omega \\
\operatorname{div} v=0, x \in \Omega \\
\int_{\sigma\left(x_{1}\right)} v_{1}=\phi,\left.\quad v_{2}^{\varepsilon}(\omega, \cdot)\right|_{\Sigma_{0,1}}=0
\end{array}\right.
$$

with Navier's condition

$$
\begin{array}{lll}
v_{1}^{\varepsilon}(\omega, \cdot)=+\varepsilon \alpha_{l}(\omega) \frac{\partial v_{1}^{\varepsilon}(\omega, \cdot)}{\partial x_{2}}, & x_{2}=0, & \alpha_{l}(\omega)=\frac{U_{l, 1}^{\infty}(\omega)}{6 \phi} \\
v_{1}^{\varepsilon}(\omega, \cdot)=-\varepsilon \alpha_{u}(\omega) \frac{\partial v_{1}^{\varepsilon}(\omega, \cdot)}{\partial x_{2}}, & x_{2}=1, & \alpha_{u}(\omega)=\frac{U_{u, 1}^{\infty}}{6 \phi}
\end{array}
$$

with $U_{l}^{\infty}, U_{u}^{\infty}$ as in theorem 3 . Note that by linearity of (2.7), constants $\alpha_{l, u}(\omega)$ depend only on the roughness, not on the flux $\phi$. We state:

\section{Theorem 5}

$$
\sup _{R \geq 1} \frac{1}{R} \mathbb{E}\left(\int_{\Omega(R)}\left|u^{\varepsilon}(\omega, \cdot)-v^{\varepsilon}(\omega, \cdot)\right|^{2}\right)=o\left(\varepsilon^{2}\right), \quad \varepsilon \rightarrow 0 .
$$

Thus, the Navier's friction law leads to a (slightly) better approximation than Dirichlet's law. Again, the proof of theorem 5 involves the ergodic theorem, which prevents quantitative bounds (the $o(\varepsilon)$ can not a priori be precised). We end this presentation of the results with two remarks:

1. All the estimates of section 2.2.2 involve the expectation of the spatial quadratic norms. Due to a lack of deterministic control of the boundary layers, we are not able to obtain almost sure estimates in $B_{2}\left(\Omega^{\varepsilon}\right)$, like in theorem 2 . However, one can deduce informations with high probability. For instance, theorem 5 and Tchebitchev inequality show that: for all $R, \delta>0$,

$$
P\left(\omega, \int_{\Omega(R)}\left|u^{\varepsilon}(\omega, \cdot)-v^{\varepsilon}(\omega, \cdot)\right|^{2}>\delta \varepsilon^{2}\right) \underset{\varepsilon \rightarrow 0}{\longrightarrow} 0 .
$$


2. Under an assumption of ergodicity on the group $\left(\tau_{h}\right)$, that is

$$
\forall A \in \mathcal{P},\left(\tau_{h}(A)=A, \forall h\right) \Rightarrow(\pi(A)=0 \text { or } \pi(A)=1),
$$

the constants $\alpha_{l, u}$ that appear in (2.12) are independent of $\omega$, as a consequence of the ergodic theorem (see section 1 ). In such case, one does not need to know the shape of the boundary to determine the appropriate coefficient in Navier's wall law. It can be deduced almost surely from a numerical computation involving another boundary.

\section{Justification of Dirichlet wall law}

\subsection{Well-posedness}

This section is devoted to the proof of theorem 1. The solution $\left(u^{\varepsilon}, p^{\varepsilon}\right)$ is searched as a perturbation of the Poiseuille type flow $\left(u^{0}, p^{0}\right)$, where $u^{0}$ is defined by (2.4), and

$$
p^{0}(\omega, x)=-12 \nu \phi x_{1}, \quad \forall x \in \Omega^{\varepsilon}(\omega) .
$$

We denote $u^{\varepsilon}=u^{0}+w, p^{\varepsilon}=p^{0}+q$, and consider the following system (dependence on $\omega$ is omitted to lighten notations):

$$
\left\{\begin{array}{l}
w \cdot \nabla w+u^{0} \cdot \nabla w+w \cdot \nabla u^{0}+\nabla q-\nu \Delta w=f^{\varepsilon}, \quad x \in \Omega^{\varepsilon} \backslash\left(\Sigma_{0} \cup \Sigma_{1}\right), \\
\operatorname{div} w=0, \quad x \in \Omega^{\varepsilon} \\
{\left.[w]\right|_{\Sigma_{0} \cup \Sigma_{1}}=0,\left.\quad\left[\partial_{2} w-q e_{2}\right]\right|_{\Sigma_{0} \cup \Sigma_{1}}=-6 \phi} \\
\int_{\sigma\left(x_{1}\right)} w_{1}=0,\left.\quad w\right|_{\partial \Omega^{\varepsilon}}=0
\end{array}\right.
$$

where

$$
\begin{aligned}
& f^{\varepsilon}(\omega, x)=0, \quad x \text { in } \Omega, \\
& f^{\varepsilon}(\omega, x)=(-12 \nu \phi, 0), \quad x \text { in } \Omega^{\varepsilon}(\omega) \backslash \Omega .
\end{aligned}
$$

The proof of theorem 1 is divided in three steps:

1. We consider the linear problem

$$
\left\{\begin{array}{l}
\left(v+u^{0}\right) \cdot \nabla w+w \cdot \nabla u^{0}+\nabla q-\nu \Delta w=f+\operatorname{div} G \\
x \in \Omega^{\varepsilon} \backslash\left(\Sigma_{0} \cup \Sigma_{1}\right), \quad \operatorname{div} w=0, \quad x \in \Omega^{\varepsilon} \\
{\left.[w]\right|_{\Sigma_{0} \cup \Sigma_{1}}=0,\left.\quad\left[\partial_{2} w-q e_{2}\right]\right|_{\Sigma_{0} \cup \Sigma_{1}}=\tilde{\phi}} \\
\int_{\sigma\left(x_{1}\right)} w_{1}=0,\left.\quad w\right|_{\partial \Omega^{\varepsilon}}=0
\end{array}\right.
$$

where for all $\omega$,

$$
\begin{aligned}
& G(\omega, \cdot) \in L_{u l o c}^{2}\left(\Omega^{\varepsilon}(\omega)\right)=\left\{G, \sup _{\tau>0} \int_{\substack{x \in \Omega_{1} \mid<\tau+1 \\
\varepsilon}}|G|^{2}<+\infty\right\}, \\
& f(\omega, \cdot) \in L_{\text {uloc }}^{2}\left(\Omega^{\varepsilon}(\omega)\right), f=0 \text { in } \Omega, \\
& v(\omega, \cdot) \in H_{\text {uloc }}^{1}\left(\Omega^{\varepsilon}(\omega)\right)=\left\{v, \quad(v, \nabla v) \in L_{u l o c}^{2}\left(\Omega^{\varepsilon}(\omega)\right)\right\}, \\
& \tilde{\phi}(\omega, \cdot) \in L_{\text {uloc }}^{2}(\mathbb{R}) .
\end{aligned}
$$


We show the existence and uniqueness of a solution

$$
w(\omega, \cdot) \in H_{u l o c}^{1}\left(\Omega^{\varepsilon}(\omega)\right), \quad \forall \omega .
$$

2. Thanks to this linear analysis, we show that, for $\varepsilon<\varepsilon_{0}, \phi<\phi_{0}$ small enough, there exists a unique solution $w$ of (1.1), thus of a solution $u^{\varepsilon}$ of (1.1), as in theorem 1 .

3. We show measurability properties of $u^{\varepsilon}$.

\subsubsection{Linear problem}

To build a solution of (3.2), we consider the following approximate problem:

$$
\left\{\begin{array}{l}
\left(v+u^{0}\right) \cdot \nabla w_{n}+w_{n} \cdot \nabla u^{0}+\nabla q_{n}-\nu \Delta w_{n}=f+\operatorname{div} G \\
x \in \Omega_{n}^{\varepsilon} \backslash\left(\Sigma_{0}(n) \cup \Sigma_{1}(n)\right), \quad \operatorname{div} w_{n}=0, \quad x \in \Omega_{n}^{\varepsilon}, \\
{\left.\left[w_{n}\right]\right|_{\Sigma_{0}(n) \cup \Sigma_{1}(n)}=0,\left.\quad\left[\partial_{2} w_{n}-q e_{2}\right]\right|_{\Sigma_{0}(n) \cup \Sigma_{1}(n)}=\tilde{\phi}} \\
\left.w_{n}\right|_{\partial \Omega_{n}^{\varepsilon}}=0,
\end{array}\right.
$$

where $\Omega_{n}^{\varepsilon}$ is a short-hand for the bounded domain $\Omega^{\varepsilon}(\omega, n)$. A formal energy estimate yields

$$
\begin{aligned}
\nu \int_{\Omega_{n}^{\varepsilon}}\left|\nabla w_{n}\right|^{2}= & \int_{\Omega_{n}^{\varepsilon} \backslash \Omega(n)} f w_{n}-\int_{\Omega_{n}^{\varepsilon}} G \nabla w_{n}-\int_{\Omega_{n}^{\varepsilon}}\left(w_{n} \cdot \nabla u^{0}\right) w_{n} \\
& +\tilde{\phi} \int_{\Sigma_{0}(n)} w_{n}-\tilde{\phi} \int_{\Sigma_{1}(n)} w_{n} \\
\leq & C\left(\sqrt{n}\|f\|_{L_{u l o c}^{2}}\left\|w_{n}\right\|_{L^{2}\left(\Omega_{n}^{\varepsilon} \backslash \Omega(n)\right)}\right. \\
& +\sqrt{n}\|G\|_{L_{u l o c}^{2}}\left\|\nabla w_{n}\right\|_{L^{2}\left(\Omega_{n}^{\varepsilon}\right)} \\
& \left.+\phi\left\|w_{n}\right\|_{L^{2}\left(\Omega_{n}^{\varepsilon}\right)}^{2}+\|\tilde{\phi}\|_{L_{u l o c}^{2}} \sqrt{n}\left\|w_{n}\right\|_{L^{2}\left(\Sigma_{0}(n) \cup \Sigma_{1}(n)\right)}\right)
\end{aligned}
$$

We remind the Poincaré inequality

$$
\|\varphi\|_{L^{2}\left(\mathcal{O}_{a}\right)} \leq C a\|\nabla \varphi\|_{L^{2}\left(\mathcal{O}_{a}\right)}
$$

valid in a domain

$$
\mathcal{O}_{a}=\left\{x, \alpha<x_{1}<\beta, \gamma_{l}\left(x_{1}\right)<x_{2}<\gamma_{u}\left(x_{1}\right)\right\}, \quad \sup \left|\gamma_{l}-\gamma_{u}\right|<a,
$$

for all $\varphi$ in $H^{1}\left(\mathcal{O}_{a}\right)$, with $\varphi=0$ on one of the boundaries $\gamma_{l, u}$. It is easily deduced from (3.4) that: for all $\varphi \in H^{1}\left(\Omega_{n}^{\varepsilon} \backslash \Omega(n)\right), \varphi=0$ on $\partial \Omega_{n}^{\varepsilon} \backslash \Omega(n)$,

$$
\begin{aligned}
& \|\varphi\|_{L^{2}\left(\Omega_{n}^{\varepsilon}-\Omega(n)\right)} \leq C \varepsilon\|\nabla \varphi\|_{L^{2}\left(\Omega_{n}^{\varepsilon}-\Omega(n)\right)}, \\
& \|\varphi\|_{L^{2}\left(\Sigma_{0}(n) \cup \Sigma_{1}(n)\right)} \leq C \varepsilon^{1 / 2}\|\nabla \varphi\|_{L^{2}\left(\Omega_{n}^{\varepsilon} \backslash \Omega(n)\right)} .
\end{aligned}
$$

We infer from (3.4), (3.6) that

$$
\begin{aligned}
\int_{\Omega_{n}^{\varepsilon}}\left|\nabla w_{n}\right|^{2} \leq & C\left(\sqrt{n}\left(\varepsilon\|f\|_{L_{u l o c}^{2}}+\|G\|_{L_{u l o c}^{2}}+\sqrt{\varepsilon}\|\tilde{\phi}\|_{L_{u l o c}^{2}}\right)\left\|\nabla w_{n}\right\|_{L^{2}\left(\Omega_{n}^{\varepsilon}\right)}\right. \\
& \left.+\phi\left\|\nabla w_{n}\right\|_{L^{2}\left(\Omega_{n}^{\varepsilon}\right)}^{2}\right)
\end{aligned}
$$


Hence, for $\phi<\phi_{0}, \varepsilon<\varepsilon_{0}$ with $\phi_{0}, \varepsilon_{0}$ small enough, we get (uniformly in the variable $\omega \in P$ )

$$
\frac{1}{\sqrt{n}}\left\|\nabla w_{n}\right\|_{L^{2}\left(\Omega_{n}^{\varepsilon}\right)} \leq C\left(\varepsilon\|f\|_{L_{u l o c}^{2}}+\|G\|_{L_{u l o c}^{2}}+\sqrt{\varepsilon}\|\tilde{\phi}\|_{L_{u l o c}^{2}}\right) .
$$

By Lax-Milgram lemma, this estimate yields existence and uniqueness of a solution $w_{n}$ of (3.3).

We now wish to let $n$ go to infinity. However, estimate (3.7) degenerates in this limit. To obtain compactness, we will follow ideas of [19] and localize estimate (3.7) in a band of width $\eta$. To do so, we multiply the first equation of $(3.3)$ by $w_{n}$, and integrate over

$$
\Omega_{n}^{\varepsilon}(R)=\Omega_{n}^{\varepsilon} \cap\left\{0<\left|x_{1}\right|<R\right\} .
$$

Proceeding as above, we get, for $\phi<\phi_{0}$ small enough,

$$
\begin{aligned}
\int_{\Omega_{n}^{\varepsilon}(R)} \nu\left|\nabla w_{n}\right|^{2} \leq & C\left(\varepsilon^{2}\|f\|_{L_{\text {uloc }}^{2}}^{2}+\|G\|_{L_{\text {uloc }}^{2}}^{2}+\varepsilon\|\tilde{\phi}\|_{L_{\text {uloc }}^{2}}^{2}\right)(R+1) \\
& +\left|\int_{\left|x_{1}\right|=R} \nu \partial_{1} w_{n} \cdot w_{n}\right|+\left|\int_{\left|x_{1}\right|=R} q_{n} w_{n, 1}\right| \\
& +\left.\left|\int_{\left|x_{1}\right|=R} v_{1}\right| u\right|^{2}|+| \int_{\left|x_{1}\right|=R} G e_{1} \cdot u \mid .
\end{aligned}
$$

We then integrate with respect to $R$, fro $\eta$ to $\eta+1$. We deduce

$$
\begin{aligned}
F(\eta) \leq & C\left(\varepsilon^{2}\|f\|_{L_{\text {uloc }}^{2}}^{2}+\|G\|_{L_{\text {uloc }}^{2}}^{2}+\varepsilon\|\tilde{\phi}\|_{L_{\text {uloc }}^{2}}^{2}\right)(\eta+1) \\
& +\left|\int_{\Omega_{n}^{\varepsilon}(\eta, \eta+1)} \partial_{1} w_{n} \cdot w_{n}\right|+\left|\int_{\Omega_{n}^{\varepsilon}(\eta, \eta+1)} q_{n} w_{n, 1}\right| \\
& +\left.\left|\int_{\Omega_{n}^{\varepsilon}(\eta, \eta+1)} v_{1}\right| w_{n}\right|^{2}|+| \int_{\Omega_{n}^{\varepsilon}(\eta, \eta+1)} G e_{1} \cdot w_{n} \mid .
\end{aligned}
$$

where

$$
F(\eta)=\int_{\eta}^{\eta+1} \nu \int_{\Omega_{n}^{\varepsilon}(R)}\left|\nabla w_{n}\right|^{2} d R, \quad \Omega_{n}^{\varepsilon}(\eta, \eta+1)=\Omega_{n}^{\varepsilon} \cap\left\{\eta<\left|x_{1}\right|<\eta+1\right\} .
$$

By Poincaré inequality (3.4), and Sobolev inequality

$$
\|\phi\|_{L^{4}\left(O_{a}\right)} \leq C\|\phi\|_{H^{1}\left(O_{a}\right)}
$$

one has easily that

$$
\begin{aligned}
& \left|\int_{\Omega_{n}^{\varepsilon}(\eta, \eta+1)} \partial_{1} w_{n} \cdot w_{n}\right| \leq C \int_{\Omega_{n}^{\varepsilon}(\eta, \eta+1)}\left|\nabla w_{n}\right|^{2}, \\
& \left.\left.\left|\int_{\Omega_{n}^{\varepsilon}(\eta, \eta+1)} v_{1}\right| w_{n}\right|^{2}\left|\leq C\|v\|_{H_{u l o c}^{1}\left(\Omega_{n}^{\varepsilon}\right)} \int_{\Omega_{n}^{\varepsilon}(\eta, \eta+1)}\right| \nabla w_{n}\right|^{2}, \\
& \left|\int_{\Omega_{n}^{\varepsilon}(\eta, \eta+1)} G e_{1} \cdot w_{n}\right| \leq C\|G\|_{L_{u l o c}^{2}\left(\Omega_{n}^{\varepsilon}\right)}\left(\int_{\Omega_{n}^{\varepsilon}(\eta, \eta+1)}\left|\nabla w_{n}\right|^{2}\right)^{1 / 2} .
\end{aligned}
$$


The treatment of the integral involving the pressure is exactly the same as in [19]. Boundary conditions and incompressibility of $w_{n}$ imply that $\int_{\left|x_{1}\right|=R} w_{n, 1}=0$ for all $R$, which yields $\int_{\Omega_{n}^{\varepsilon}(\eta, \eta+1)} w_{n, 1}=0$. It is then well-known (see 13] for detailed description) that: there exists

$$
\varphi_{\eta} \in H_{0}^{1}\left(\Omega_{n}^{\varepsilon}(\eta, \eta+1)\right), \quad \text { with } \operatorname{div} \varphi_{\eta}=w_{1},
$$

and

$$
\left\|\varphi_{\eta}\right\|_{H_{0}^{1}\left(\Omega_{n}^{\varepsilon}(\eta, \eta+1)\right)} \leq C\left\|w_{1}\right\|_{L^{2}\left(\Omega_{n}^{\varepsilon}(\eta, \eta+1)\right)}
$$

where $C$ is a positive constant independent on $\varepsilon, n$ and $\eta$. We can write

$$
\int_{\Omega_{n}^{\varepsilon}(\eta, \eta+1)} q_{n} w_{n, 1}=\int_{\Omega_{n}^{\varepsilon}(\eta, \eta+1)} q_{n} \operatorname{div} \varphi_{\eta}=-\int_{\Omega_{n}^{\varepsilon}(\eta, \eta+1)} \nabla q_{n} \cdot \varphi_{\eta} .
$$

Using the expression of $\nabla q_{n}$ in (3.3), and after several integrations by parts,

$$
\begin{aligned}
\left|\int_{\Omega_{n}^{\varepsilon}(\eta, \eta+1)} q_{n} w_{n, 1}\right| \leq & \left|\int_{\Omega_{n}^{\varepsilon}(\eta, \eta+1)} \nu \nabla w_{n} \cdot \nabla \varphi_{\eta}\right|+\left|\int_{\Omega_{n}^{\varepsilon}(\eta, \eta+1)}\left(v+u^{0}\right) \cdot \nabla w_{n} \varphi_{\eta}\right| \\
& +\left|\int_{\Omega_{n}^{\varepsilon}(\eta, \eta+1)} \nabla \varphi_{\eta} w_{n} \cdot u^{0}\right|+\left|\int_{\Omega_{n}^{\varepsilon}(\eta, \eta+1)}(f+\operatorname{div} G) w_{n}\right|
\end{aligned}
$$

This leads to the inequality

$$
\begin{aligned}
\left|\int_{\Omega_{n}^{\varepsilon}(\eta, \eta+1)} q_{n} w_{n, 1}\right| \leq & C\left(1+\|v\|_{H_{u l o c}^{1}}\right) \int_{\Omega_{n}^{\varepsilon}(\eta, \eta+1)} \mid \nabla w_{n} \|^{2} \\
& +C\left(\varepsilon^{2}\|f\|_{L_{u l o c}^{2}}^{2}+\|G\|_{L_{u l o c}^{2}}^{2}\right) .
\end{aligned}
$$

Together with previous bounds, this yields

$$
\begin{aligned}
F(\eta) \leq & C\left(1+\|v\|_{H_{u l o c}^{1}}\right) \int_{\Omega_{n}^{\varepsilon}(\eta, \eta+1)} \mid \nabla w_{n} \|^{2} \\
& +C\left(\varepsilon^{2}\|f\|_{L_{u l o c}^{2}}^{2}+\|G\|_{L_{u l o c}^{2}}^{2}+\varepsilon\|\tilde{\phi}\|_{L_{u l o c}^{2}}^{2}\right)(1+\eta) .
\end{aligned}
$$

that is

$$
F(\eta) \leq \mathcal{C}_{1} F^{\prime}(\eta)+\mathcal{C}_{2}(1+\eta)
$$

where

$$
\mathcal{C}_{1}=C_{1}\left(1+\|v\|_{H_{u l o c}^{1}}\right), \quad \mathcal{C}_{2}=C_{2}\left(\varepsilon^{2}\|f\|_{L_{\text {uloc }}^{2}}^{2}+\|G\|_{L_{\text {uloc }}^{2}}^{2}+\varepsilon\|\tilde{\phi}\|_{L_{\text {uloc }}^{2}}^{2}\right) .
$$

This last equation is a reverse Gronwall type inequality. By estimate (3.7), up to take a larger $C_{2}$, we can suppose that

$$
F(n) \leq \mathcal{C}_{2}\left(\mathcal{C}_{1}+n+1\right) .
$$

From (3.10), we deduce easily that for all $\eta$,

$$
\begin{aligned}
& e^{-\frac{1}{\mathcal{C}_{1}} n} F(n)-e^{-\frac{1}{\mathcal{C}_{1}} \eta} F(\eta) \geq-\mathcal{C}_{2} \int_{\eta}^{n} \frac{(t+1)}{\mathcal{C}_{1}} e^{-\frac{1}{\mathcal{C}_{1}} t} d t \\
& \geq \mathcal{C}_{2}\left(\mathcal{C}_{1}+n+1\right) e^{-\frac{1}{\mathcal{C}_{1}} n}-\mathcal{C}_{2}\left(\mathcal{C}_{1}+\eta+1\right) e^{-\frac{1}{\mathcal{C}_{1}} \eta} .
\end{aligned}
$$


It follows that for all $\eta$,

$$
F(\eta) \leq \mathcal{C}\left(\varepsilon^{2}\|f\|_{L_{\text {uloc }}^{2}}^{2}+\|G\|_{L_{u l o c}^{2}}^{2}+\varepsilon\|\tilde{\phi}\|_{L_{u l o c}^{2}}^{2}\right)(\eta+1)
$$

and finally

$$
\sup _{\eta \geq 1} \frac{1}{\eta} \int_{\Omega_{n}^{\varepsilon}(\eta)}\left|\nabla w_{n}\right|^{2} \leq \mathcal{C}\left(\varepsilon^{2}\|f\|_{L_{\text {uloc }}^{2}}^{2}+\|G\|_{L_{\text {uloc }}^{2}}^{2}+\varepsilon\|\tilde{\phi}\|_{L_{\text {uloc }}^{2}}^{2}\right)
$$

where $\mathcal{C}$ can be taken affine in $\|v\|_{H_{u l o c}^{1}}$.

On the basis of such estimate, one can, for all $\omega$, extract a subsequence $w_{\phi_{\omega}(n)}$ that converges weakly in $H_{l o c}^{1}\left(\Omega^{\varepsilon}(\omega)\right)$. The limit $w(\omega, \cdot)$ is a solution of (3.2), and satisfies the estimate

$$
\sup _{\eta \geq 1} \frac{1}{\eta} \int_{\Omega_{n}^{\varepsilon}(\eta)}|\nabla w|^{2} \leq \mathcal{C}\left(\varepsilon^{2}\|f\|_{L_{u l o c}^{2}}^{2}+\|G\|_{L_{u l o c}^{2}}^{2}+\varepsilon\|\tilde{\phi}\|_{L_{u l o c}^{2}}^{2}\right) .
$$

It remains to show that $w$ is in $H_{u l o c}^{1}$. The argument is almost the same as in [19, p745]. Let $\tau>3 / 2$. By energy estimates carried on the translated domain

$$
\Omega^{\varepsilon}(\tau, R)=\Omega^{\varepsilon}(R)+(\tau, 0),
$$

we obtain similarly to (3.10)

$$
F_{\tau}(\eta) \leq \mathcal{C}_{1} F_{\tau}^{\prime}(\eta)+\mathcal{C}_{2}(1+\eta), \quad F_{\tau}(\eta)=\int_{\eta}^{\eta+1} \int_{\Omega^{\varepsilon}(\tau, R)}|\nabla w|^{2} d R .
$$

By (3.12), we have

$$
F_{\tau}(\tau) \leq \mathcal{C}\left(\varepsilon^{2}\|f\|_{L_{\text {uloc }}^{2}}^{2}+\|G\|_{L_{\text {uloc }}^{2}}^{2}+\varepsilon\|\tilde{\phi}\|_{L_{\text {uloc }}^{2}}^{2}\right)(\tau+1),
$$

and reasoning as above, we get: for all $0<\eta<\tau$,

$$
F_{\tau}(\eta)<\mathcal{C}\left(\varepsilon^{2}\|f\|_{L_{u l o c}^{2}}^{2}+\|G\|_{L_{\text {uloc }}^{2}}^{2}+\varepsilon\|\tilde{\phi}\|_{L_{\text {uloc }}^{2}}^{2}\right)(\eta+1) .
$$

Applying this inequality with $\eta=3 / 2$ yields

$$
\|w\|_{H_{u l o c}^{1}}^{2} \leq \mathcal{C}\left(\varepsilon^{2}\|f\|_{L_{u l o c}^{2}}^{2}+\|G\|_{L_{u l o c}^{2}}^{2}+\varepsilon\|\tilde{\phi}\|_{L_{u l o c}^{2}}^{2}\right)
$$

For uniqueness, one must show that the solution $w$ of (3.2) with $f=0, G=0, \tilde{\phi}=0$ is identically zero. Inequality (3.10) turns into

$$
F(\eta) \leq \mathcal{C}_{1} F^{\prime}(\eta), \quad \forall \eta>0
$$

from which it follows easily that

$$
\limsup _{\eta \rightarrow+\infty} F(\eta) e^{-\mathcal{C}_{1} \eta} \geq F(0) .
$$

Together with (3.12), we deduce $w=0$. Note that uniqueness does not only hold in $H_{\text {uloc }}^{1}\left(\Omega^{\varepsilon}(\omega)\right)$ but in the wider space $B^{2}\left(\Omega^{\varepsilon}(\omega)\right)$, for any $\omega \in P$. 


\subsubsection{Nonlinear problem}

This section is devoted to the well-posedness of (3.1), for any fixed $\omega \in P$. Therefore, we consider linear equations (3.2) with special choice

$$
f=f^{\varepsilon}, G=0, \text { and } \tilde{\phi}=-6 \phi .
$$

Note that $\left\|f^{\varepsilon}\right\|_{L_{u l o c}^{2}} \leq C \sqrt{\varepsilon}$. We define the application

$$
\Lambda: H_{u l o c}^{1}\left(\Omega^{\varepsilon}(\omega)\right) \mapsto H_{u l o c}^{1}\left(\Omega^{\varepsilon}(\omega)\right), \quad v(\omega, \cdot) \mapsto w(\omega, \cdot),
$$

with $w(\omega, \cdot)$ the solution of (3.2). Then, for $\varepsilon<\varepsilon_{0}$ small enough, $\Lambda$ is a contraction in restriction to the unit ball of $H_{u l o c}^{1}\left(\Omega^{\varepsilon}(\omega)\right)$, .

Indeed, let $v^{1}, v^{2}$ in the unit ball of $H_{u l o c}^{1}\left(\Omega^{\varepsilon}(\omega)\right)$, and denote $w^{1}=\Lambda\left(v^{1}\right), w^{2}=\Lambda\left(v^{2}\right)$. By estimate (3.14), they are bounded by

$$
\left\|w^{1,2}\right\|_{H_{u l o c}^{1}} \leq C\left(\varepsilon^{2}\left\|f^{\varepsilon}\right\|_{L_{u l o c}^{2}}^{2}+\varepsilon\|\phi\|_{L_{u l o c}^{2}}^{2}\right) \leq C \varepsilon
$$

for $\varepsilon<\varepsilon_{0}$ small enough. The difference $w=w^{1}-w^{2}$ satisfies (3.2), with

$$
v=v^{1}, f=0, G=\left(v^{2}-v^{1}\right) \otimes w^{2}, \tilde{\phi}=0 .
$$

Again, using (3.14) leads to

$$
\begin{aligned}
\|w\|_{H_{u l o c}^{1}} & \leq C\left\|\left(v^{2}-v^{1}\right) \otimes w^{2}\right\|_{L_{u l o c}^{2}} \leq C\left\|v^{2}-v^{1}\right\|_{H_{u l o c}^{1}}\left\|w^{2}\right\|_{H_{u l o c}^{1}} \\
& \leq C \sqrt{\varepsilon}\left\|v^{2}-v^{1}\right\|_{H_{u l o c}^{1}} .
\end{aligned}
$$

Hence, for $\varepsilon<\varepsilon_{0}$ small enough, $\Lambda$ is a contraction in restriction to the unit ball. By the Banach fixed point theorem, we deduce the existence of a unique solution $w(\omega, \cdot)$ of $(3.1)$ in the unit ball of $H_{u l o c}^{1}\left(\Omega^{\varepsilon}(\omega)\right)$, for all $\omega \in P$. Back to the original variables, there exists a solution $u^{\varepsilon}(\omega, \cdot)$ of (1.1), which is unique in the ball of center $u^{0}$ and radius one in $H_{u l o c}^{1}\left(\Omega^{\varepsilon}(\omega)\right)$.

Uniqueness in the space $B_{2}\left(\Omega^{\varepsilon}(\omega)\right)$ is deduced easily from [19, theorem 2.3, p739]. The idea is still to obtain local estimates on the difference of two solutions. Due to the quadratic term in Navier-Stokes equations, the inequality (3.15) is modified by a nonlinear term, but still leads to uniqueness. We do not give further details, and refer to [19].

\subsubsection{Measurability}

To conclude the proof of theorem 1, it remains to check the measurability properties of $\omega \mapsto \tilde{u}^{\varepsilon}(\omega, \cdot), P \mapsto H_{l o c}^{1}\left(\mathbb{R}^{2}\right)$, where the $\sim$ stands for the extension by zero outside $\Omega^{\varepsilon}$. We know from the previous section that $w=u^{\varepsilon}-u^{0}$ is the fixed point of a contraction. Precisely, for all $\omega \in P, w(\omega, \cdot)$ is the strong limit in $H_{u l o c}^{1}\left(\Omega^{\varepsilon}(\omega)\right)$ of the sequence $\left(w^{k}(\omega, \cdot)\right)_{k \in \mathbb{N}}$, satisfying

$$
\left\{\begin{array}{l}
w^{k} \cdot \nabla w^{k+1}+u^{0} \cdot \nabla w^{k+1}+w^{k+1} \cdot \nabla u^{0}+\nabla q^{k+1}-\nu \Delta w^{k+1}=f^{\varepsilon}, \\
x \in \Omega^{\varepsilon} \backslash\left(\Sigma_{0} \cup \Sigma_{1}\right), \quad \operatorname{div} w^{k+1}=0, \quad x \in \Omega^{\varepsilon}, \\
{\left.\left[w^{k+1}\right]\right|_{\Sigma_{0} \cup \Sigma_{1}}=0,\left.\quad\left[\partial_{2} w-q e_{2}\right]\right|_{\Sigma_{0} \cup \Sigma_{1}}=-6 \phi} \\
\int_{\sigma\left(x_{1}\right)} w_{1}^{k+1}=0,\left.\quad w^{k+1}\right|_{\partial \Omega^{\varepsilon}}=0,
\end{array}\right.
$$


in which we take $\left\|w^{0}\right\|_{H_{u l o c}^{1}} \leq 1$, with $\omega \mapsto \tilde{w}^{0}(\omega, \cdot)$ measurable. Thus, it is enough to show that for all $k, \omega \mapsto \tilde{w}^{k}(\omega, \cdot)$ is measurable from $P$ to $H_{l o c}^{1}\left(\mathbb{R}^{2}\right)$. Therefore, we will prove that for any function

$$
v(\omega, \cdot) \in H_{u l o c}^{1}\left(\Omega^{\varepsilon}(\omega)\right), \quad \omega \mapsto \tilde{v}(\omega, \cdot) \text { measurable, }
$$

the solution $w$ of (3.2) (with $\left.f=f^{\varepsilon}, G=0, \tilde{\phi}=-6 \phi\right)$ is such that $\omega \mapsto \tilde{w}(\omega, \cdot)$ is measurable.

With previous arguments and notations, for all $\omega$, there is a subsequence $w_{\phi_{\omega}(n)}(\omega, \cdot)$ of $w_{n}(\omega, \cdot)$ that converges weakly in $H_{l o c}^{1}\left(\omega^{\varepsilon}(\omega)\right)$ to $w(\omega, \cdot)$. By uniqueness of $w$ as a solution of (3.3), one can easily check that the whole sequel $w_{n}(\omega, \cdot)$ converges to $w(\omega, \cdot)$. Hence, by Pettis theorem [23], it is enough to show the measurability of $\omega \mapsto \tilde{w}_{n}(\omega, \cdot)$.

To do so, we follow ideas of [1] on an elliptic problem from homogeneization. Let us introduce

$$
\left.C_{n}=\right]-n, n[\times]-2,2[,
$$

and the spaces

$$
\begin{aligned}
V_{n} & =\left\{w \in H_{0}^{1}\left(C_{n}\right), \operatorname{div} w=0\right\}, \\
V_{n}(\omega) & =\left\{w \in V_{n}, w=0 \text { in } C_{n} \backslash \Omega^{\varepsilon}(\omega, n)\right\} .
\end{aligned}
$$

Let $\pi_{n}(\omega): V_{n} \mapsto V_{n}$ the orthogonal projection on $V_{n}(\omega)$. By definition of $(P, \mathcal{P})$, it is easy to show that for all $n$, the set-valued map

$$
\mathcal{F}_{n}:(P, \mathcal{P}) \mapsto \mathcal{P}_{c}\left(H^{1}\left(\mathbb{R}^{2}\right)\right), \quad \omega \mapsto V_{n}(\omega),
$$

is measurable. Following [10], we remind that a set-valued map

$$
\mathcal{F}:(P, \mathcal{P}) \mapsto \mathcal{P}_{c}(X), \quad \omega \mapsto \mathcal{F}(\omega),
$$

from a measurable space $P$ to the non-empty complete subsets of a separable metric space $X$ is measurable if: for all open subset $\mathcal{O}$ of $X$, the set

$$
\{\omega, \mathcal{F}(\omega) \cap \mathcal{O} \neq \emptyset\}
$$

belongs to $\mathcal{P}$ (in our case, this set is open, and so belongs to the $\sigma$-algebra $\mathcal{P}$ ). For all $w$ in $V_{n}$, there exists by property (3.18) a sequence of measurable selections $\sigma_{j}(\omega)$ such that $\sigma_{j}(\omega) \rightarrow \pi_{n}(\omega) w$ strongly in $V_{n}$ (c.f. [10, theorem III.9, p67]). We thus get, for all $w, w^{\prime}$ in $V_{n}$,

$$
\left(\left(\pi_{n}(\omega) u, v\right)\right)=\lim _{j}\left(\left(\sigma_{j}(\omega), w^{\prime}\right)\right),
$$

where $((\cdot, \cdot))$ denotes the usual scalar product on $H_{0}^{1}\left(C_{n}\right)$. Thus, for all $w, w^{\prime}$,

$$
\omega \mapsto\left(\left(\pi_{n}(\omega) w, w^{\prime}\right)\right)
$$

is measurable, which by Pettis theorem yields the measurability of $\pi_{n}$.

Assume now that $\omega \mapsto \tilde{v}(\omega, \cdot)$ is measurable. There exists $\alpha_{n}>0$, such that for all $\omega$, the solution $w_{n}(\omega, \cdot)$ of (3.2) is the fixed point of the contraction:

$$
F(\omega): V_{n} \mapsto V_{n}, \quad w \mapsto \pi_{n}(\omega)\left(\alpha_{n}(A(\omega) w-l(\omega))+w\right),
$$


where $l(\omega) \in V_{n}$, and $A(\omega): V_{n} \mapsto V_{n}$ are defined by

$$
\begin{aligned}
\left(\left(l(\omega), w^{\prime}\right)\right)= & 6 \phi \int_{\Sigma_{0}(n)} w^{\prime}-6 \phi \int_{\Sigma_{1}(n)} w^{\prime}+\int_{C_{n}} \tilde{f}^{\varepsilon}(\omega, \cdot) w^{\prime} \\
\left(\left(A(\omega) w, w^{\prime}\right)\right)= & \nu \int_{C_{n}}\left(\nabla w \cdot \nabla w^{\prime}+\left(\tilde{v}(\omega, \cdot)+\tilde{u}^{0}(\omega, \cdot)\right) \cdot \nabla w \cdot w^{\prime}\right. \\
& \left.+w \cdot \nabla u^{0}(\omega, \cdot) \cdot w^{\prime}\right)
\end{aligned}
$$

Thus, $w_{n}(\omega, \cdot)$ is obtained as the limit of the sequence

$$
w_{n}^{j+1}=F(\omega)\left(w_{n}^{j}\right)
$$

It is clear that $\omega \mapsto l(\omega)$ and $\omega \mapsto A(\omega)$ are measurable. Together, with the measurability of $\pi_{n}(\omega)$, it follows easily that $\omega \mapsto F(\omega) w$ is measurable for all $w$ in $H^{1}\left(\mathbb{R}^{2}\right)$, hence the application $\omega \mapsto \tilde{w}_{n}^{j}(\omega, \cdot)$, from $P$ to $H^{1}\left(\mathbb{R}^{2}\right)$, for all $j$. As $\tilde{w}_{n}$ is the limit of $\tilde{w}_{n}^{j}$, theorem $\square$ is proved.

\subsection{Estimates for Dirichlet wall law}

We now turn to the proof of theorem 2. Instead of estimates in $B_{2}$ norm, we will show the following more precise $L_{u l o c}^{2}$ estimates, valid for $\phi$ small enough:

$$
\begin{aligned}
& \|\nabla w\|_{L_{u l o c}^{2}\left(\Omega^{\varepsilon}\right)} \leq C \sqrt{\varepsilon} \\
& \|w\|_{L_{u l o c}^{2}\left(\Sigma_{0,1}\right)} \leq C \varepsilon \\
& \|w\|_{L_{u l o c}^{2}(\Omega)} \leq C \varepsilon .
\end{aligned}
$$

In fact, the first of these inequalities is just (3.14) with $G=0$, and the second one is an easy consequence of (3.6). So we just need to focus on the third inequality, which will be proved through a duality argument. The main task is to estimate $\|w\|_{L^{2}\left(\Omega_{R}\right)}$ (where $\left.\Omega_{R}=\Omega \cap\left\{-R<x_{1}<R\right\}\right)$. In $\Omega$, we can see $w$ as the solution of a modified Stokes system, with inhomogeneous Dirichlet conditions on $\Sigma_{0}$ and $\Sigma_{1}$ :

$$
\left\{\begin{array}{l}
\nabla q-\nu \Delta w=-\left(w+u^{0}\right) \cdot \nabla w-w \cdot \nabla u^{0}, \quad x \in \Omega \\
\operatorname{div} w=0, \quad x \in \Omega \\
w \text { given on } \Sigma_{0,1}
\end{array}\right.
$$

the boundary values of $w$ being controlled by (3.20). We introduce the adjoint equations in $\Omega_{R}$, that is the Stokes system with vanishing boundary conditions and non-zero source term:

$$
\left\{\begin{array}{l}
\nabla \pi-\nu \Delta v=\varphi, \quad x \in \Omega_{R} \\
\operatorname{div} v=0, \quad x \in \Omega_{R} \\
\left.v\right|_{\partial \Omega_{R}}=0
\end{array}\right.
$$

where $\varphi \in L^{2}\left(\Omega_{R}\right)$. We proceed in two steps. First, we establish two regularity lemmas for (3.23). Then, by appropriate choice of $\varphi$, we obtain the claimed estimate (3.21). 


\subsubsection{Two lemmas for the Stokes system}

It is well known from the elliptic regularity theory that the solution $(v, \pi)$ of $(3.23)$ lies in $H^{2} \times H^{1}$ as soon as $\varphi \in L^{2}$, and that there exists $C\left(\Omega_{R}\right)$ such that $\|v\|_{H^{2}\left(\Omega_{R}\right)}+\|\pi\|_{H^{1}\left(\Omega_{R}\right)} \leq$ $C\left(\Omega_{R}\right)\|\varphi\|_{L^{2}\left(\Omega_{R}\right)}$ (here $\pi$ is the zero-mean determination of the pressure). However, the constant $C\left(\Omega_{R}\right)$ depends a priori on $R$. We need an estimate independent of $R$, which may be obtained by localization.

Lemma 6 There exists $C>0$ such that for all $R>1$, the solution $(v, \pi)$ of (3.23) satisfies

$$
\|v\|_{H^{2}\left(\Omega_{R}\right)}+\|\nabla \pi\|_{L^{2}\left(\Omega_{R}\right)} \leq C\|\varphi\|_{L^{2}\left(\Omega_{R}\right)} .
$$

Proof.

Let $\chi \in C^{\infty}(\mathbb{R})$ such that $\chi=1$ on $[0,1], \chi=0$ outside $[-1,2]$. Let $\chi_{k}\left(x_{1}\right)=\chi\left(x_{1}-k\right)$. We will also make use of two other truncation functions $\theta_{L}$ et $\theta_{R}$ such that $\theta_{L} \in C^{\infty}(\mathbb{R})$, $\theta_{L}=1$ on $\left.]-\infty, 2\right], \theta_{L}=0$ on $\left[3, \infty\left[\right.\right.$, and $\theta_{R}\left(x_{1}\right)=\theta_{L}\left(-x_{1}\right)$. Let $K=\lfloor R\rfloor-1$. We localize the equations thanks to the functions $\theta_{L}, \chi_{k}$ and $\theta_{R}$ : let $v_{k}\left(x_{1}, x_{2}\right)=\chi_{k}\left(x_{1}\right) v\left(x_{1}, x_{2}\right)$ $(-K \leq k \leq K-1), v_{L}=\theta_{L}\left(x_{1}+R\right) v, v_{R}=\theta_{R}\left(x_{1}-R\right) v$. The function $v_{k}$ (resp. $\left.v_{L}, v_{R}\right)$ vanishes outside the domain $D_{k}=(k-1, k+2) \times(0,1)\left(\right.$ resp. $D_{L}=(-R,-R+3) \times(0,1)$, $\left.D_{R}=(R-3, R) \times(0,1)\right)$. Let $c_{k}$ be the mean of the pressure $\pi$ on $D_{k}$. We set $\pi_{k}=\chi_{k}\left(\pi-c_{k}\right)$. We define similarly $\pi_{L}, \pi_{R}$. The functions $v_{k}, \pi_{k}$ solve the following equations in $D_{k}$ :

$$
\left\{\begin{array}{l}
\nabla \pi_{k}-\nu \Delta v_{k}=\varphi_{k}, \quad x \in D_{k} \\
\operatorname{div} v_{k}=\gamma_{k}, \quad x \in D_{k} \\
\left.v_{k}\right|_{\partial D_{k}}=0,
\end{array}\right.
$$

where

$$
\begin{gathered}
\varphi_{k}=\chi_{k} \varphi-\nu\left(2 \nabla \chi_{k} \cdot \nabla v+v \cdot \Delta \chi_{k}\right)+\nabla \chi_{k}\left(\pi-c_{k}\right), \\
\gamma_{k}=\nabla \chi_{k} \cdot v .
\end{gathered}
$$

In $D_{L}$ and $D_{R}, v_{L}$ and $v_{R}$ solve analogous systems (note that $v_{L}$ equals zero on the left boundary of $D_{L}$, for $v$ does). The classical elliptic theory applied to this system yields

$$
\left\|v_{k}\right\|_{H^{2}\left(D_{k}\right)}^{2}+\left\|\nabla \pi_{k}\right\|_{L^{2}\left(D_{k}\right)}^{2} \leq C\left(\left\|\varphi_{k}\right\|_{L^{2}\left(D_{k}\right)}^{2}+\left\|\gamma_{k}\right\|_{H^{1}\left(D_{k}\right)}^{2}\right) .
$$

Of course, the constant $C$ is independent of $k$. We have

$$
\begin{aligned}
\sum_{k=-K}^{K-1}\left\|\varphi_{k}\right\|_{L^{2}\left(D_{k}\right)}^{2}+\left\|\gamma_{k}\right\|_{H^{1}\left(D_{k}\right)}^{2} \leq & C\left(\|\varphi\|_{L^{2}\left(\Omega_{R}\right)}^{2}+\|v\|_{H^{1}\left(\Omega_{R}\right)}^{2}\right) \\
& +C \sum_{k}\left\|\pi-c_{k}\right\|_{L^{2}\left(D_{k}\right)}^{2} .
\end{aligned}
$$

We can control $\pi-c_{k}$ in the following way. One can find $C>0$ such that for all $f \in L^{2}\left(D_{k}\right)$ with $\int_{D_{k}} f=0$, there exists a function $\psi \in H_{0}^{1}\left(D_{k}\right)$ satisfying $\operatorname{div} \psi=f$ and $\|\psi\|_{H_{0}^{1}\left(D_{k}\right)} \leq$ $C\|f\|_{L^{2}\left(D_{k}\right)}$. Hence we have for such $f$ and $\psi$,

$$
\begin{aligned}
\left|\int_{D_{k}}\left(\pi-c_{k}\right) f\right| & =\left|\int_{D_{k}} \nabla \pi \cdot \psi\right| \\
& \leq\|\nabla \pi\|_{H^{-1}\left(D_{k}\right)}\|\psi\|_{H_{0}^{1}\left(D_{k}\right)} \\
& \leq C\|\nabla \pi\|_{H^{-1}\left(D_{k}\right)}\|f\|_{L^{2}\left(D_{k}\right)} .
\end{aligned}
$$


Thus we obtain

$$
\left\|\pi-c_{k}\right\|_{L^{2}\left(D_{k}\right)} \leq C\|\nabla \pi\|_{H^{-1}\left(D_{k}\right)} \leq C\left(\|\varphi\|_{L^{2}\left(D_{k}\right)}+\|\nabla v\|_{L^{2}\left(D_{k}\right)}\right) .
$$

We have similar results for $v_{L}$ and $v_{R}$. Noticing that on $(k, k+1) \times(0,1)$, we have $v_{k}=v$ and $\nabla \pi_{k}=\nabla p$, and similarly with $v_{L}, v_{R}$ on $(-R,-R+2) \times(0,1)$ and $(R-2, R) \times(0,1)$, the previous inequalities lead to

$$
\begin{aligned}
\|v\|_{H^{2}\left(\Omega_{R}\right)}^{2}+\|\nabla \pi\|_{L^{2}\left(\Omega_{R}\right)}^{2} & \leq \sum_{k=-K-1}^{K}\left(\left\|v_{k}\right\|_{H^{2}\left(D_{k}\right)}^{2}+\left\|\nabla \pi_{k}\right\|_{L^{2}\left(D_{k}\right)}^{2}\right) \\
& \leq C\left(\|\varphi\|_{L^{2}\left(\Omega_{R}\right)}^{2}+\|v\|_{H^{1}\left(\Omega_{R}\right)}^{2}\right) \\
& \leq C\|\varphi\|_{L^{2}\left(\Omega_{R}\right)}^{2}
\end{aligned}
$$

(where indices $k=-K-1$ and $k=K$ stand for $k=L$ and $k=K$ to lighten notations). Last bound comes from Poincaré inequality and from the standard energy estimate of (3.23) in the whole domain $\Omega_{R}$. This ends the proof of the lemma. set

Our second lemma is an $H_{u l o c}^{1}$ estimate for the Stokes system in $\Omega_{R}$, uniform in $R$. We

$$
\|v\|_{L_{u l o c}^{2}\left(\Omega_{R}\right)}^{2}=\sup _{-R<\tau<R-1} \int_{(\tau, \tau+1) \times(0,1)}|v|^{2}
$$

and $\|v\|_{H_{u l o c}^{1}\left(\Omega_{R}\right)}^{2}=\|v\|_{L_{u l o c}^{2}\left(\Omega_{R}\right)}^{2}+\|\nabla v\|_{L_{u l o c}^{2}\left(\Omega_{R}\right)}^{2}$.

Lemma 7 There exists a constant $C>0$ such that for all $R>1$, we have

$$
\|v\|_{H_{u l o c}^{1}\left(\Omega_{R}\right)} \leq C\|\varphi\|_{L_{u l o c}^{2}\left(\Omega_{R}\right)} .
$$

Proof.

The proof is very similar to the calculations which led to the estimate (3.14), so we only sketch it briefly. We write an energy estimate on the domain $(-R, r) \times(0,1)$, and integrate with respect to $r$, from $\eta$ to $\eta+1$, to obtain a backward Gronwall type inequality:

$$
F(\eta) \leq C \int_{(-R, \eta+1) \times(0,1)}|\varphi|^{2}+C F^{\prime}(\eta) \leq C\|\varphi\|_{L_{u l o c}^{2}}^{2}(R+\eta+1)+C F^{\prime}(\eta)
$$

$(-R \leq \eta \leq R-1)$, where

$$
F(\eta)=\int_{\eta}^{\eta+1} \int_{(-R, r) \times(0,1)}|\nabla v|^{2} d x d r .
$$

For $\eta=R-1$, we have

$$
F(R-1) \leq\|\nabla v\|_{L^{2}\left(\Omega_{R}\right)}^{2} \leq\|\varphi\|_{L^{2}\left(\Omega_{R}\right)}^{2} \leq 2 R\|\varphi\|_{L_{\text {uloc }}^{2}}^{2},
$$

thus we obtain

$$
F(\eta) \leq C\|\varphi\|_{L_{u l o c}^{2}}^{2}(R+\eta+1) .
$$

Similarly, we have, for $-R \leq \eta \leq R-1$,

$$
G(\eta)=\int_{\eta}^{\eta+1} \int_{(r, R) \times(0,1)}|\nabla v|^{2} d x d r \leq C\|\varphi\|_{L_{u l o c}^{2}}^{2}(R-\eta) .
$$


Now let $\tau \in[-R+1, R-1]$. Again, an energy estimate on $(\tau-r, \tau+r) \times(0,1)$ leads to

$$
H_{\tau}(\eta)=\int_{\eta}^{\eta+1} \int_{(\tau-r, \tau+r) \times(0,1)}|\nabla v|^{2} d x d r \leq C\|\varphi\|_{L_{u l o c}^{2}}^{2}(\eta+1)+C H_{\tau}^{\prime}(\eta)
$$

$(0 \leq \eta \leq R-1-|\tau|)$. If, for instance, $\tau>0$, we have

$$
\begin{aligned}
H_{\tau}(R-1-\tau) & \leq \int_{(2 \tau-R, R) \times(0,1)}|\nabla v|^{2} \\
& \leq G(2 \tau-R-1) \\
& \leq C\|\varphi\|_{L_{\text {uloc }}^{2}}^{2}(2 R-2 \tau+1),
\end{aligned}
$$

hence we conclude that for all $0 \leq \eta \leq R-1-\tau$,

$$
H_{\tau}(\eta) \leq C\|\varphi\|_{L_{u l o c}^{2}}^{2}(\eta+1) .
$$

If $\tau<0$, we obtain a similar conclusion using $F$ instead of $G$. For $\eta=1 / 2$, we get

$$
\int_{\left(\tau-\frac{1}{2}, \tau+\frac{1}{2}\right) \times(0,1)}|\nabla v|^{2} \leq C\|\varphi\|_{L_{u l o c}^{2}}^{2}
$$

The result follows.

\subsubsection{The duality estimate}

Now we estimate $w$ using a duality method. Let $R>0$, and let $v^{R}, \pi^{R}$ denote the solution of the Stokes problem (3.23) in $\Omega_{R}$ with source term $\varphi=\left.w\right|_{\Omega_{R}}$. Using these auxiliary functions, let us show the following inequality for $\phi$ small enough:

$$
\int_{\Omega_{R}}|w|^{2} \leq C R \varepsilon^{2}+C \int_{\left|x_{1}\right|=R}|w|^{2} d x_{2}
$$

To prove this, our starting point will be:

$$
\begin{aligned}
\int_{\Omega_{R}}|w|^{2} & =\int_{\Omega_{R}} w \cdot\left(-\nu \Delta v^{R}+\nabla \pi^{R}\right) \\
& =\int_{\Omega_{R}}\left(-\nu \Delta w \cdot v^{R}+w \cdot \nabla \pi^{R}\right)-\int_{\partial \Omega_{R}} \nu w \cdot \frac{\partial v^{R}}{\partial n} .
\end{aligned}
$$

We have to control the various terms appearing in (3.26). We recall the equation satisfied by $w$ in $\Omega_{R}$ :

$$
-\nu \Delta w+(w \cdot \nabla)\left(w+u^{0}\right)+u_{1}^{0} \partial_{1} w+\nabla q=0 .
$$

Thus integrations by parts lead to

$$
\begin{aligned}
\left|\int_{\Omega_{R}} \nu \Delta w \cdot v^{R}\right| & =\left|\int_{\Omega_{R}}-w \cdot(w \cdot \nabla) v^{R}+v^{R} \cdot(w \cdot \nabla) u^{0}-u_{1}^{0} w \cdot \partial_{1} v^{R}\right| \\
& \leq \int_{\Omega_{R}}|w|^{2}\left|\nabla v^{R}\right|+C \phi\|w\|_{L^{2}\left(\Omega_{R}\right)}\left\|v^{R}\right\|_{H^{1}\left(\Omega_{R}\right)}
\end{aligned}
$$


Let $\left(a_{0}, \ldots, a_{N}\right)$ be a regular subdivision of the interval $[-R, R]$, such that $a_{0}=-R, a_{N}=R$, and $1 \leq a_{k+1}-a_{k} \leq 2$ (hence $N \leq 2 R$ ). We divide the domain $\Omega_{R}$ into the corresponding cells $C_{k}=\left(a_{k-1}, a_{k}\right) \times(0,1)$. Then we can write, using the Gagliardo-Nirenberg inequality in each cell $C_{k}$,

$$
\begin{aligned}
\int_{\Omega_{R}}|w|^{2}\left|\nabla v^{R}\right| & \leq \sum_{k=1}^{N}\|w\|_{L^{4}\left(C_{k}\right)}^{2}\left\|\nabla v^{R}\right\|_{L^{2}\left(C_{k}\right)} \\
& \leq \sum_{k} C\|w\|_{L^{2}\left(C_{k}\right)}\|w\|_{H^{1}\left(C_{k}\right)}\left\|\nabla v^{R}\right\|_{L_{u l o c}^{2}} \\
& \leq \sum_{k}\left(\eta\|w\|_{L^{2}\left(C_{k}\right)}^{2}+C(4 \eta)^{-1}\|w\|_{H_{u l o c}^{1}}^{2}\left\|v^{R}\right\|_{H_{u l o c}^{1}}^{2}\right) \\
& \leq \eta\|w\|_{L^{2}\left(\Omega_{R}\right)}^{2}+C R \eta^{-1}\|w\|_{H_{u l o c}^{1}}^{4},
\end{aligned}
$$

thanks to the second lemma, with $\eta$ a small parameter to be chosen later. We obtain, using the energy inequality for (3.23), estimate (3.19) and the previous inequalities (3.27), (3.28):

$$
\left|\int_{\Omega_{R}} \nu \Delta w \cdot v^{R}\right| \leq(\eta+C \phi)\|w\|_{L^{2}\left(\Omega_{R}\right)}^{2}+C R \eta^{-1} \varepsilon^{2} .
$$

Next, we treat the boundary integral in (3.26). We have, for $i=0,1$, thanks to (3.20) and lemma 6,

$$
\begin{aligned}
\left|\int_{\Sigma_{i}(R)} \nu w \cdot \partial_{2} v^{R} d x_{1}\right| & \leq \nu\|w\|_{L^{2}\left(\Sigma_{i}(R)\right)}\left\|\nabla v^{R}\right\|_{L^{2}\left(\Sigma_{i}(R)\right)} \\
& \leq C \varepsilon \sqrt{R}\left\|v^{R}\right\|_{H^{2}\left(\Omega_{R}\right)} \\
& \leq C \eta^{-1} \varepsilon^{2} R+\eta\|w\|_{L^{2}\left(\Omega_{R}\right)}^{2}
\end{aligned}
$$

We also write, using again a trace theorem,

$$
\begin{aligned}
\left|\int_{\left|x_{1}\right|=R} \nu w \cdot \partial_{1} v^{R} d x_{2}\right| & \leq C\left(\int_{\left|x_{1}\right|=R}|w|^{2} d x_{2}\right)^{\frac{1}{2}}\left\|v^{R}\right\|_{H^{1}\left(\Omega_{R-1, R}\right)} \\
& \leq C \eta^{-1} \int_{\left|x_{1}\right|=R}|w|^{2} d x_{2}+\eta\|w\|_{L^{2}\left(\Omega_{R}\right)}^{2}
\end{aligned}
$$

(here the integrals on the sections $x_{1}= \pm R$ are restricted to the portion of each section contained in $\Omega$ ). It remains to deal with the integral involving the pressure $\pi^{R}$ in (3.26). We use again the subdivision of $\Omega_{R}$ into the cells $C_{k}$. Let $c_{k}$ denote the mean of $\pi^{R}$ on $C_{k}$. We integrate by parts $w \cdot \nabla \pi^{R}=w \cdot \nabla\left(\pi^{R}-c_{k}\right)$ on each cell $C_{k}$ :

$$
\begin{aligned}
\int_{\Omega_{R}} w \cdot \nabla \pi^{R}= & \sum_{k} \int_{\partial C_{k}} w \cdot n\left(\pi^{R}-c_{k}\right) \\
= & \sum_{k}\left(\int_{\Sigma_{1} \cap \partial C_{k}} w_{2}\left(\pi^{R}-c_{k}\right) d x_{1}-\int_{\Sigma_{0} \cap \partial C_{k}} w_{2}\left(\pi^{R}-c_{k}\right) d x_{1}\right) \\
& -\int_{x_{1}=-R} w_{1}\left(\pi^{R}-c_{1}\right) d x_{2}+\int_{x_{1}=R} w_{1}\left(\pi^{R}-c_{N}\right) d x_{2} \\
& +\sum_{k=1}^{n-1} \int_{x_{1}=a_{k}} w_{1}\left(c_{k+1}-c_{k}\right) d x_{2} .
\end{aligned}
$$


We bound the quantities appearing in the r.h.s. of (3.32). We have, for $i=0,1$,

$$
\begin{aligned}
\sum_{k}\left|\int_{\Sigma_{i} \cap \partial C_{k}} w_{2}\left(\pi^{R}-c_{k}\right) d x_{1}\right| & \leq \sum_{k} C\|w\|_{L_{u l o c}^{2}\left(\Sigma_{i}\right)}\left\|\pi^{R}-c_{k}\right\|_{H^{1}\left(C_{k}\right)} \\
& \leq \sum_{k}\left(C \eta^{-1} \varepsilon^{2}+\eta\left\|\nabla \pi^{R}\right\|_{L^{2}\left(C_{k}\right)}\right) \\
& \leq C \eta^{-1} \varepsilon^{2} R+\eta\|w\|_{L^{2}\left(\Omega_{R}\right)}^{2} .
\end{aligned}
$$

using (3.20), the Poincaré-Wirtinger inequality applied to the zero-mean function $\pi^{R}-c_{k}$ in $C_{k}$, and lemma 6. Similarly, we have for the two terms corresponding to $x_{1}= \pm R$ :

$$
\left|\int_{x_{1}= \pm R} w^{1}\left(\pi^{R}-c_{1, N}\right) d x_{2}\right| \leq C \eta^{-1} \int_{\left|x_{1}\right|=R}|w|^{2} d x_{2}+\eta\|w\|_{L^{2}\left(\Omega_{R}\right)}^{2} .
$$

To deal with the last term of (3.32), we use the fact that $\int_{\sigma\left(a_{k}\right)} w_{1} d x_{2}=0$, where $\sigma\left(a_{k}\right)=$ $\left\{\left(a_{k}, x_{2}\right) \in \Omega^{\varepsilon}\right\}$ (here we take into account the exterior $\Omega^{\varepsilon} \backslash \Omega$ of $\Omega$ ). Hence

$$
\begin{aligned}
\left|\int_{x_{1}=a_{k}} w_{1} d x_{2}\right| & =\left|\int_{\sigma\left(a_{k}\right) \backslash \Omega} w_{1} d x_{2}\right| \\
& \leq\left(\varepsilon \int_{\sigma\left(a_{k}\right) \backslash \Omega}\left|w_{1}\right|^{2} d x_{2}\right)^{1 / 2} \\
& \leq C \sqrt{\varepsilon}\|w\|_{H_{u l o c}^{1}\left(\Omega^{\varepsilon}\right)}
\end{aligned}
$$

(we can apply the trace theorem in $\Omega^{\varepsilon} \cap\left\{a_{k}<x_{1}<a_{k+1}\right\}$ for instance, with a constant independent of $k$ since the boundaries of $\Omega^{\varepsilon}$ are $K$-Lipschitz continuous). Moreover, we obviously have $\left|c_{k+1}-c_{k}\right| \leq C\left\|\nabla \pi^{R}\right\|_{L^{2}\left(C_{k} \cup C_{k+1}\right)}$, thus estimate (3.19) and lemma 6 again imply

$$
\begin{aligned}
\left|\sum \int_{x_{1}=a_{k}} w_{1}\left(c_{k+1}-c_{k}\right) d x_{2}\right| & \leq \sum C \varepsilon\left\|\nabla \pi^{R}\right\|_{L^{2}\left(C_{k} \cup C_{k+1}\right)} \\
& \leq C \eta^{-1} \varepsilon^{2} R+\eta\|w\|_{L^{2}\left(\Omega_{R}\right)}^{2} .
\end{aligned}
$$

Up to this point, the r.h.s. of (3.32) can be controlled as follows:

$$
\left|\int_{\Omega_{R}} w \cdot \nabla \pi^{R}\right| \leq C \eta^{-1} \varepsilon^{2} R+4 \eta\|w\|_{L^{2}\left(\Omega_{R}\right)}^{2}+C \int_{\left|x_{1}\right|=R}|w|^{2} d x_{2} .
$$

Then inequalities (3.29), (3.30), (3.31) and (3.33) enable us to write, from (3.26):

$$
\int_{\Omega_{R}}|w|^{2} \leq(C \phi+8 \eta)\|w\|_{L^{2}\left(\Omega_{R}\right)}^{2}+C R \eta^{-1} \varepsilon^{2}+C \eta^{-1} \int_{\left|x_{1}\right|=R}|w|^{2} d x_{2}
$$

We take $\eta=1 / 10$ and $\phi<\phi_{0}$ such that $C \phi_{0}<1 / 10$. This leads to (3.25).

Let $z(R)=\|w\|_{L^{2}\left(\Omega_{R}\right)}^{2}$. Inequality (3.25) can be rewritten $z(R) \leq \mathcal{C} R \varepsilon^{2}+\mathcal{C} z^{\prime}(R)$. Following [19], we deduce the estimate (2.5) from this differential inequality and from the fact that $z(t)$ has subexponential growth at infinity (Poincaré inequality and (3.19) imply that it has linear growth). Indeed, let $\tilde{z}(R)=\mathcal{C} R \varepsilon^{2}+\mathcal{C}^{2} \varepsilon^{2}$; we have $z^{\prime}(R)-\tilde{z}^{\prime}(R) \geq \frac{z(R)-\tilde{z}(R)}{\mathcal{C}}$. Hence if 
there existed $R_{0}$ such that $z\left(R_{0}\right)-\tilde{z}\left(R_{0}\right)=A>0$, we would have $z(R)-\tilde{z}(R) \geq A e^{\left(R-R_{0}\right) / \mathcal{C}}$ for all $R \geq R_{0}$, and $z$ would grow exponentially fast at infinity, which is not the case. Thus we obtain for all $R>1, z(R) \leq \mathcal{C} R \varepsilon^{2}+\mathcal{C}^{2} \varepsilon^{2}$, that is (2.5).

Finally, we show (3.21). It is a direct consequence of what we have already proved. For $R=1$, we have $\|w\|_{L^{2}\left(\Omega_{1}\right)}^{2}=z(1) \leq C \varepsilon^{2}$. Now we can repeat the whole proof but with another origin: take $x_{1}=\tau$ instead of $x_{1}=0$ as origin, replace $\Omega_{R}$ with $\Omega(\tau, R)=\Omega_{R}+(\tau, 0)$. This yields $z_{\tau}(R)=\|w\|_{L^{2}(\Omega(\tau, R))}^{2} \leq \mathcal{C} R \varepsilon^{2}+\mathcal{C}^{2} \varepsilon^{2}$, with the same constant $\mathcal{C}$ as before. Hence we have again $\|w\|_{L^{2}(\Omega(\tau, 1))}^{2} \leq C \varepsilon^{2}$, and we conclude that $\|w\|_{L_{u l o c}^{2}(\Omega)}^{2} \leq C \varepsilon^{2}$.

\section{The boundary layer analysis}

\subsection{Formal expansion}

To obtain a refined wall law at $\partial \Omega$, one must clarify the structure of the flow near the rough boundary. Namely, we will show that $u^{\varepsilon}$ behaves as

$$
\begin{aligned}
u^{\varepsilon}(\omega, x) \approx u_{\text {app }}^{\varepsilon}(\omega, x)= & u^{0}(\omega, x)+\varepsilon U_{l}\left(\omega, \frac{x_{1}}{\varepsilon}, \frac{x_{2}}{\varepsilon}\right)+\varepsilon U_{u}\left(\omega, \frac{x_{1}}{\varepsilon}, \frac{x_{2}-1}{\varepsilon}\right) \\
& +\varepsilon u^{1}(\omega, x) .
\end{aligned}
$$

In this formal expansion, $U_{l, u}(\omega, y)$ are boundary layer profiles, expressing the strong gradients of $u^{\varepsilon}$ near the boundary. They are defined on the rescaled domains $\mathcal{R}_{l, u}(\omega)$. They should be localized near the boundary, and cancel the jump of the normal derivative of $u^{0}$. As usual, to derive the system they satisfy, we plug expansions (4.1) in equations (1.1), and collect terms of order $\varepsilon^{-1}$. Formally, this leads to the Stokes system (2.7)a,b. Jump conditions (2.7)c,d ensure the regularity of the approximation through $\partial \Omega$. Finally, (2.7) $\mathrm{d}$ is in agreement with the Dirichlet condition (1.2).

As mentioned in theorem 3, we will show that $U_{l, u}(\omega, \cdot)$ converge to some constant fields

$$
U_{l, u}^{\infty}(\omega)=\left(U_{l, u, 1}^{\infty}(\omega), 0\right)
$$

as $y_{2} \rightarrow+\infty$. Because of these fields, which do not match the required flux and boundary conditions, there is an additional corrector $u^{1}(\omega, x)$. It is given by

$$
\left\{\begin{array}{l}
u^{1}(\omega, x)=\left(3\left(U_{l, 1}^{\infty}+U_{u, 1}^{\infty}\right) x_{2}^{2}-\left(4 U_{l, 1}^{\infty}+2 U_{u, 1}^{\infty}\right) x_{2}-U_{u, 1}^{\infty}, 0\right), \quad x \in \Omega, \\
u^{1}(\omega, x)=-U_{u}^{\infty}, \quad x_{2}<0 \\
u^{1}(\omega, x)=-U_{l}^{\infty}, \quad x_{2}>1 .
\end{array}\right.
$$

Note that in the interior domain $\Omega, u^{1}$ satisfies

$$
\left\{\begin{array}{l}
u^{0} \cdot \nabla u^{1}+u^{1} \cdot \nabla u^{0}-\nu \Delta u^{1}+\nabla p^{1}=0, \quad x \in \Omega, \\
\operatorname{div} u^{1}=0, \quad x \in \Omega, \\
\left.u^{1}\right|_{\Sigma_{0}}=-\left.U_{u}^{\infty} \quad u^{1}\right|_{\Sigma_{1}}=-U_{l}^{\infty}, \quad \int_{\sigma\left(x_{1}\right)} u_{1}^{1}=-\left(U_{l, 1}^{\infty}+U_{u, 1}^{\infty}\right) .
\end{array}\right.
$$




\subsection{Well-posedness of (2.7)}

We now justify the previous formal computations, and prove theorem 3 . We first consider the well-posedness of system (2.7). In the study of Dirichlet wall law, the Poincaré inequality (3.4), applied to the channel $\Omega^{\varepsilon}$, allowed for deterministic reasoning. In the boundary layer domains $\mathcal{R}_{l, u}(\omega)$ that are unbounded in every direction, this inequality does not hold, and we shall use the probabilistic modeling to solve (2.7). This approach borrows to homogeneization problems (see [18, 7, 8]), but strong changes are needed to account for the anisotropy of our boundary layer domains. The general idea is to construct a solution of the type

$$
U_{l, u}(\omega, y)=V_{l, u}\left(\tau_{y_{1}}(\omega), y_{2} \pm h_{l, u} \circ \tau_{y_{1}}(\omega)\right), \quad V_{l, u}=V_{l, u}(\omega, \lambda),
$$

where $\tau_{y_{1}}$ and $h_{l, u}$ were defined in subsection 2.1. This requires to introduce adapted functional spaces and variational formulations. As the lower and upper systems are treated in the same way, we will focus on the lower one, and drop the subscript "l" for brevity.

\subsubsection{Stochastic derivative, Convolution}

Let $V: P \times \mathbb{R} \mapsto \mathbb{R}^{n}, n \geq 1$, measurable. We call a realization of $V$ an application

$$
R_{\omega}[V]: \mathbb{R}^{2} \mapsto \mathbb{R}^{n}, \quad y \mapsto V\left(\tau_{y_{1}}(\omega), y_{2}+h \circ \tau_{y_{1}}(\omega)\right), \quad \omega \in P .
$$

Notice that

$$
R_{\tau_{h}}(\omega)[V]\left(y_{1}, y_{2}\right)=R_{\omega}[V]\left(y_{1}+h, y_{2}\right), \quad \forall h, y .
$$

We say that $V$ is smooth if, almost surely, $R_{\omega}[V]$ is smooth as a function on $\mathbb{R}^{2}$. For all smooth functions $V$, we define the stochastic derivative of $V$ as

$$
\partial_{\omega} V(\omega, \lambda)=\partial_{1} R_{\omega}[V]\left(y_{1}=0, y_{2}=\lambda-h(\omega)\right)
$$

We also introduce

$$
\partial_{\lambda} V(\omega, \lambda)=\partial_{2} R_{\omega}[V]\left(y_{1}=0, y_{2}=\lambda-h(\omega)\right)
$$

and the gradient $\nabla_{\omega}=\left(\partial_{\omega}, \partial_{\lambda}\right)$. Notice that, almost surely,

$$
R_{\omega}\left[\nabla_{\omega} V\right]=\nabla R_{\omega}[V]
$$

which allows to define for smooth $V$ partial derivatives at any order $\partial_{\omega}^{\alpha} \partial_{\lambda}^{\beta} V$.

For interesting examples of smooth functions, one can use a convolution process. Precisely, let $\rho=\rho(y) \in \mathbb{R}$ a smooth function with compact support. For all $V \in L_{l o c}^{1}(P \times \mathbb{R})=$ $\cap_{K} L^{1}(P \times K), K$ compact, we define

$$
\rho * V(\omega, \lambda)=\int_{\mathbb{R}^{2}} \rho(y) V\left(\tau_{y_{1}}(\omega), \lambda+y_{2}+h \circ \tau_{y_{1}}(\omega)-h(\omega)\right) d y .
$$

The following lemma is easy and left to the reader.

Lemma 8 Assume $1 \leq p \leq \infty$ and $V \in L^{p}(P \times \mathbb{R})$, resp. $L_{l o c}^{p}$. Then $\rho * V \in L^{p}(P \times \mathbb{R})$, resp. $L_{l o c}^{p}$, and is a smooth function. Moreover, the following identities hold:

$$
\begin{aligned}
& R_{\omega}[\rho * V]=\hat{\rho} * R_{\omega}[V], \text { a.s. in } \omega, \\
& \nabla_{\omega}(\rho * V)=\nabla \hat{\rho} * V,
\end{aligned}
$$

where $\hat{\rho}(y)=\rho(-y)$. Besides, if $V$ is smooth and $\nabla_{\omega} V \in L_{\text {loc }}^{1}(P \times \mathbb{R})$, then

$$
\nabla_{\omega}(\rho * V)=\rho * \nabla_{\omega} V \text {. }
$$




\subsubsection{Functional spaces}

We can now introduce functional spaces adapted to (2.7). Let $\mathcal{D}_{0}\left(P \times \mathbb{R}_{+}\right)$the set of smooth functions $\varphi=\varphi(\omega, \lambda) \in \mathbb{R}^{2}$, satisfying

i) For all $\alpha, \beta, \quad \partial_{\omega}^{\alpha} \partial_{\lambda}^{\beta} \varphi \in L^{\infty}(P \times \mathbb{R})$.

ii) Almost surely, the support of $R_{\omega}[\varphi]$ is included in $\left\{y, \frac{1}{\delta}>y_{2}+h \circ \tau_{y_{1}}(\omega)>\delta\right\}$ for some positive constant $\delta=\delta(\omega, \varphi)$.

Note that realizations of functions of $\mathcal{D}_{0}\left(P \times \mathbb{R}_{+}\right)$have a support that is strictly included in $\mathcal{R}(\omega)$, almost surely in $\omega$. We clearly define a scalar product on $\mathcal{D}_{0}\left(P \times \mathbb{R}_{+}\right)$through

$$
((\varphi, \tilde{\varphi}))=\int_{P \times \mathbb{R}} \nabla_{\omega} \varphi \cdot \nabla_{\omega} \tilde{\varphi}
$$

Let $D_{0}\left(P \times \mathbb{R}_{+}\right)$the completion of $\mathcal{D}_{0}\left(P \times \mathbb{R}_{+}\right)$for the norm $\|\varphi\|_{D_{0}}=((\varphi, \varphi))^{\frac{1}{2}}$. It is of course a Hilbert space. We state the following properties:

Lemma 9 We have

i) $D_{0}\left(P \times \mathbb{R}_{+}\right) \hookrightarrow C^{0}\left(\mathbb{R}_{+} ; L^{2}(P)\right) \hookrightarrow L_{l o c}^{2}\left(P \times \mathbb{R}_{+}\right)$.

ii) For any $V$ in $D_{0}\left(P \times \mathbb{R}_{+}\right)$, there exists a unique $D_{\omega} V \in L^{2}\left(P \times \mathbb{R}_{+}\right)$such that

$$
\int_{P \times \mathbb{R}_{+}}\left(D_{\omega} V\right) \varphi=-\int_{P \times \mathbb{R}_{+}}\left(\nabla_{\omega} \varphi\right) V, \quad \forall \varphi \in \mathcal{D}_{0}\left(P \times \mathbb{R}_{+}\right) .
$$

Moreover, $\left\|D_{\omega} V\right\|=\|V\|_{D_{0}}$. With a slight abuse of notation, we shall write $\nabla_{w}=$ $\left(\partial_{\lambda}, \partial_{\omega}\right)$ instead of $D_{\omega}$ ("weak stochastic derivative").

iii) For any $V$ in $D_{0}\left(P \times \mathbb{R}_{+}\right)$, almost surely, $R_{\omega}[V] \in H_{\text {loc }}^{1}(\mathcal{R}(\omega)),\left.R_{\omega}[V]\right|_{\partial(\mathcal{R}(\omega))}=0$, $\nabla R_{\omega}[V]=R_{\omega}\left[\nabla_{\omega} V\right]$

Proof.

i) We can argue for $V \in \mathcal{D}_{0}$, as the result for $D_{0}$ follows by density. Almost surely, $R_{\omega}[V]$ belongs to $H_{\text {loc }}^{1}\left(\mathcal{R}(\omega),\left.\quad R_{\omega}[V]\right|_{\partial \mathcal{R}(\omega)}=0\right.$. Thus,

$$
\begin{aligned}
\int_{P}|V|^{2}(\omega, \lambda) d P & =\frac{1}{2} \int_{(-1,1)} d y_{1} \int_{P}|V|^{2}\left(\tau_{y_{1}}(\omega), \lambda\right) d P \\
& =\frac{1}{2} \int_{P}\left\|R_{\omega}[V]\left(y_{1}, \lambda-h \circ \tau_{y_{1}}(\omega)\right)\right\|_{L^{2}(-1,1)}^{2} d P \\
& \leq C(\lambda) \int_{P}\left\|\nabla R_{\omega}[V](x, z)\right\|_{L^{2}(\mathcal{R}(\omega, 1))}^{2} d P \\
& =C(\lambda) \int_{P}\left\|R_{\omega}\left[\nabla_{\omega} V\right](x, z)\right\|_{L^{2}(\mathcal{R}(\omega, 1))}^{2} d P \\
& =2 C(\lambda) \int_{P \times \mathbb{R}_{+}}\left|\nabla_{\omega} V\right|^{2} d P d \lambda
\end{aligned}
$$

where the inequality comes from classical trace theorem and Poincaré inequality. 
ii) Let $V \in D_{0}$, and $V_{n} \in \mathcal{D}_{0}$, converging to $V$. As $\left\|V_{n}-V_{m}\right\|_{D_{0}}=\left\|\nabla_{\omega} V_{n}-\nabla_{\omega} V_{m}\right\|, \nabla_{\omega} V_{n}$ is a Cauchy sequence in $L^{2}\left(P \times \mathbb{R}_{+}\right)$, thus converging to some $D_{\omega} V$ with $\left\|D_{\omega} V\right\|_{L^{2}}=\|V\|_{D_{0}}$. As

$$
\int_{P \times \mathbb{R}_{+}}\left(\nabla_{\omega} V_{n}\right) \varphi=-\int_{P \times \mathbb{R}_{+}}\left(\nabla_{\omega} \varphi\right) V_{n}, \quad \forall \varphi \in \mathcal{D}_{0}\left(P \times \mathbb{R}_{+}\right),
$$

relation (4.6) follows (notice that the right hand side converges by i)).

It remains to show uniqueness of $D_{\omega} V$. This follows easily from the following general result: for any $W$ in $L^{p}\left(P \times \mathbb{R}_{+}\right)$, resp. $L_{l o c}^{p}, 1 \leq p<\infty$, there exists $W_{n} \in \mathcal{D}_{0}\left(P \times \mathbb{R}_{+}\right)$such that

$$
W_{n} \underset{n \rightarrow+\infty}{\longrightarrow} W \quad \text { in } L^{p}\left(P \times \mathbb{R}_{+}\right) \text {, resp. } L_{l o c}^{p} .
$$

To see this, first notice that functions of $L^{\infty}\left(P \times \mathbb{R}_{+}\right)$with compact support in $\lambda$ are dense in $L^{p}\left(P \times \mathbb{R}_{+}\right)$, resp. $L_{l o c}^{p}$. We therefore assume that $W$ is such a function. We then proceed by convolution: let $\rho_{n}$ an approximation of unity, and set $W_{n}=\rho_{n} * W$. From lemma 8 , we deduce easily that $W_{n} \in \mathcal{D}_{0}\left(P \times \mathbb{R}_{+}\right)$. To obtain the convergence, we involve as above the realizations of $W_{n}$ and $W$, by extra integration with respect to $y_{1}$. Namely, for $W \in L^{p}$,

$$
\begin{aligned}
\int_{P \times \mathbb{R}} d P & \left|W_{n}-W\right|^{p}=\frac{1}{2} \int_{(-1,1)} d y_{1} \int_{P \times \mathbb{R}} d P\left|W_{n}-W\right|^{p} \\
& \leq \frac{1}{2} \int_{P} \int_{(-1,1) \times \mathbb{R}} d y\left|R_{\omega}\left[\rho_{n} * W\right](y)-R_{\omega}[W](y)\right|^{p} \\
& \leq \int_{P} d P\left(\int_{(-1,1) \times \mathbb{R}} d y\left|\rho_{n} * R_{\omega}[W](y)-R_{\omega}[W](y)\right|^{p}\right) .
\end{aligned}
$$

By standard results on the (standard) convolution, the integral inside the parenthesis converges to 0 as $n \rightarrow+\infty$, almost surely. The dominated convergence theorem allows to conclude. Similar reasoning holds for $W \in L_{l o c}^{p}$, with $P \times K, K$ compact, instead of $P \times \mathbb{R}$.

iii) follows again from a density argument, and is left to the reader

We finally introduce the (closed) space of divergence-free vector fields:

$$
V_{0}\left(P \times \mathbb{R}_{+}\right)=\left\{V \in D_{0}\left(P \times \mathbb{R}_{+}\right), \nabla_{\omega} \cdot V=\partial_{\omega} V_{1}+\partial_{\lambda} V_{2}=0\right\} .
$$

\subsubsection{Variational formulation}

The keypoint to solve (2.7) is to search the solution $U(\omega, \cdot)$ as a realization: almost surely,

$$
U(\omega, \cdot)=R_{\omega}[V], \quad V \in V_{0}\left(P \times \mathbb{R}_{+}\right) .
$$

At a formal level, if we substitute (4.7) in (2.7), and test again $R_{\omega}[\varphi], \varphi \in V_{0}\left(P \times \mathbb{R}_{+}\right)$, we end up with

$$
\nu \int_{P \times \mathbb{R}_{+}} \nabla_{\omega} V: \nabla_{\omega} \varphi=\mathcal{L}(\varphi), \quad \forall \varphi \in V_{0}\left(P \times \mathbb{R}_{+}\right),
$$

where the linear form

$$
\mathcal{L}(\varphi)=6 \phi \cdot \int_{P} \varphi(\omega,+h(\omega)) d P
$$

comes from the inhomogeneous jump condition on the normal derivative. Note that $\mathcal{L}$ is welldefined and continuous on $D_{0}\left(P \times \mathbb{R}_{+}\right)$by lemma 9 . This suggests the following definition: 
$U$ is a variational solution of (2.7) if it satisfies (4.7), (4.8).

Notice that by Riesz theorem, there exists a unique solution $V$ to (4.8), and so a unique variational solution $U$ to (2.7). From lemma 9, one has almost surely,

$$
U(\omega, \cdot) \in H^{1}(\mathcal{R}(\omega)),\left.\quad U(\omega, \cdot)\right|_{\partial(\mathcal{R}(\omega))}=0, \quad \nabla U(\omega, \cdot) \in L^{2}(\mathcal{R}(\omega, R)), \forall R,
$$

with for all $R \geq 1$,

$$
\frac{1}{2 R} \mathbb{E}\left(\int_{\mathcal{R}(\cdot, R)}|\nabla U(\cdot, y)|^{2} d y\right)=\left\|\nabla_{\omega} V\right\|_{L^{2}}^{2}<\infty .
$$

Moreover, by the ergodic theorem, we have

$$
\begin{aligned}
& \lim _{R \rightarrow+\infty} \frac{1}{R} \int_{\mathcal{R}(\omega, R)}|\nabla U(\cdot, y)|^{2} d y \\
= & \lim _{R \rightarrow+\infty} \frac{1}{R} \int_{(-R, R)} d y \int_{\mathbb{R}_{+}}\left|\nabla_{\omega} V\left(\tau_{y}(\omega), \lambda\right)\right|^{2} d \lambda
\end{aligned}
$$

exists almost surely, so that

$$
\sup _{R \geq 1} \frac{1}{R} \int_{\mathcal{R}(\omega, R)}|\nabla U(\cdot, y)|^{2} d y<\infty \quad \text { almost surely . }
$$

It remains to show that $U$ is almost surely a classical solution. This is a consequence of Lemma 10 Almost surely, div $U=0$ in the weak sense, and for every $\psi \in \mathcal{C}_{c}^{\infty}(\mathcal{R}(\omega))$ with div $\psi=0$, we have

$$
\nu \int_{\mathcal{R}(\omega)} \nabla U: \nabla \psi=6 \phi \int_{\Sigma_{0}} \psi\left(y_{1}, 0\right) d y
$$

Proof. By lemma 9, iii), div $U=0$ in the weak sense almost surely. To recover the Stokes equation in the weak sense, we reexpress the variational equation (4.8) as

$$
\nu \int_{P \times \mathbb{R}_{+}} \nabla_{\omega} V: \nabla_{\omega} \varphi+\int_{P \times \mathbb{R}_{+}} \nabla_{\omega} W: \nabla_{\omega} \varphi=\mathcal{L}(\varphi), \quad \forall \varphi \in D_{0}\left(P \times \mathbb{R}_{+}\right),
$$

where $W \in V_{0}^{\perp}\left(P \times \mathbb{R}_{+}\right)$, which means that

$$
\int_{P \times \mathbb{R}_{+}} \nabla_{\omega} W: \nabla_{\omega} \tilde{V}=0, \quad \forall \tilde{V} \in V_{0}\left(P \times \mathbb{R}_{+}\right) .
$$

We consider again an approximation of unity $\rho_{n}=\rho_{n}(y)$, and set $\tilde{V}=\rho_{n} *\left(\nabla_{\omega}^{\perp} \varphi\right), \varphi \in$ $\mathcal{D}_{0}\left(P \times \mathbb{R}_{+}\right)$. We compute

$$
\begin{aligned}
0 & =\int_{P \times \mathbb{R}_{+}} \nabla_{\omega} W: \nabla_{\omega}\left(\rho_{n} * \nabla_{\omega}^{\perp} \varphi\right)=\int_{P \times \mathbb{R}_{+}} \nabla_{\omega} W:\left(\rho_{n} *\left(\nabla_{\omega} \nabla_{\omega}^{\perp} \varphi\right)\right) \\
& =\int_{P \times \mathbb{R}_{+}}\left(\hat{\rho}_{n} *\left(\nabla_{\omega} W\right)\right): \nabla_{\omega} \nabla_{\omega}^{\perp} \varphi=-\int_{P \times \mathbb{R}_{+}}\left(\nabla_{\omega}^{\perp} \cdot\left(\nabla_{\omega}\right)^{2}\left(\hat{\rho}_{n} * W\right)\right) \varphi .
\end{aligned}
$$


so that $\nabla_{\omega}^{\perp} \cdot\left(\nabla_{\omega}\right)^{2}\left(\hat{\rho}_{n} * W\right)=0$. Applying $R_{\omega}$ we deduce that

$$
\nabla^{\perp} \cdot \Delta\left(\rho_{n} * R_{\omega}[W]\right)=0 .
$$

Back to (4.8), we take $\varphi_{n}=\rho_{n} * \varphi$ as a test function. One has easily the following identities:

$$
\begin{aligned}
& \int \nabla_{\omega} V: \nabla_{\omega} \varphi_{n}=-\int\left(\left(\nabla_{\omega}\right)^{2}\left(\hat{\rho}_{n} * V\right)\right) \cdot \varphi \\
& \int \nabla_{\omega} W: \nabla_{\omega} \varphi_{n}=-\int\left(\left(\nabla_{\omega}\right)^{2}\left(\hat{\rho}_{n} * W\right)\right) \cdot \varphi \\
& \mathcal{L}\left(\varphi_{n}\right)=6 \phi \cdot \int\left(\hat{\rho}_{n} *((\omega, \lambda) \mapsto \delta(\lambda-h(\omega)))\right) \cdot \varphi,
\end{aligned}
$$

where $\delta$ stands for the Dirac measure, i.e.

$$
\hat{\rho}_{n} *((\omega, \lambda) \mapsto \delta(\lambda+h(\omega)))=\int_{\mathbb{R}} d y_{1} \hat{\rho}_{n}\left(y_{1},-\lambda+h(\omega)\right) d y_{1} .
$$

We thus deduce from (4.8) that,

$$
-\nu\left(\nabla_{\omega}\right)^{2}\left(\hat{\rho}_{n} * V\right)-\left(\nabla_{\omega}\right)^{2}\left(\hat{\rho}_{n} * W\right)=6 \phi \cdot \hat{\rho}_{n} *((\omega, \lambda) \mapsto \delta(\lambda-h(\omega))),
$$

which implies that, almost surely:

$$
-\nu \Delta\left(\rho_{n} * U\right)(\omega, \cdot)-\Delta\left(\rho_{n} * R_{\omega}[W]\right)(\omega, \cdot)=6 \phi \cdot\left(\rho_{n} * \delta_{y_{2}=0}\right) .
$$

From (4.9), we can express

$$
\Delta\left(\rho_{n} * R_{\omega}[W]\right)(\omega, \cdot)=\nabla p_{\omega},
$$

for some smooth scalar field $p_{\omega}$. Hence, if we multiply (4.10) by a divergence free vector field $\psi \in \mathcal{C}_{c}^{\infty}(\mathcal{R}(\omega))$ and perform integration by parts, we obtain

$$
\int_{\mathcal{R}(\omega)} \nabla\left(\rho_{n} * U\right): \nabla \psi=\frac{\phi}{6} \int_{\Sigma_{0}}\left(\rho_{n} * \psi\right)\left(y_{1}, 0\right) d y_{1} .
$$

The limit $n \rightarrow+\infty$ gives the result. The fact that $U(\omega . \cdot)$ is regular follows from standard ellipticity properties of the Stokes operator. This shows the well-posedness of the boundary layer system (2.7).

\subsection{Convergence at infinity}

This section is devoted to the last part of theorem 3, that is convergence of $U_{l, u}$ as $y_{2} \rightarrow+\infty$. In the periodic setting, the convergence is exponential, as can be seen easily from a Fourier analysis. Namely, if the roughness has period $T$, it is straightforward that

$$
\left|U_{l, u}\left(y_{1}, y_{2}\right)-U_{l, u}^{\infty}\right| \leq C e^{-\frac{\alpha}{T}}
$$

for some constant $\alpha$ independent of $T$. In this case, $U_{l, u}^{\infty}=\left(U_{l, u, 1}^{\infty}, 0\right)$ is just the average of $U_{l, u}$ with respect to $y_{1}$. We stress that the convergence rate $\alpha / T$ goes to zero as the period of the roughness $T$ goes to infinity. Thus, in the random setting (in which, broadly 
speaking, all periods are involved in the roughness), this analysis falls down. We will show the convergence using ergodicity properties of $U_{l, u}$. For the sake of brevity, we will only treat the lower boundary layer $U_{l}$. Similar reasoning holds for the upper one.

We first establish a representation formula for $U_{l}$, in terms of the double layer Stokes potential.

Lemma 11 Almost surely, the solution $U_{l}$ of (2.7) satisfies, for $y_{2}>0$,

$$
U_{l}(\omega, y)=\int_{\mathbb{R}} G\left(y_{1}-y_{1}^{\prime}, y_{2}\right) U_{l}\left(\omega, y_{1}^{\prime}, 0\right) d y_{1}^{\prime},
$$

where

$$
G\left(y_{1}, y_{2}\right)=\frac{2 y_{2}}{\pi\left(y_{1}^{2}+y_{2}^{2}\right)^{2}}\left(\begin{array}{cc}
y_{1}^{2} & y_{1} y_{2} \\
y_{1} y_{2} & y_{2}^{2}
\end{array}\right)
$$

Proof.

Let us denote by $\tilde{U}_{l}(\omega, y)$ the right hand side of (4.11). We remind that

$$
U_{l}(\omega, y)=V\left(\tau_{y_{1}}(\omega), y_{2}+h_{l} \circ \tau_{y_{1}}(\omega)\right)=\mathcal{V}\left(\tau_{y_{1}}(\omega), y_{2}\right)
$$

As a consequence,

$$
\tilde{U}_{l}(\omega, y)=\tilde{\mathcal{V}}\left(\tau_{y_{1}}(\omega), y_{2}\right)
$$

where

$$
\tilde{\mathcal{V}}\left(\omega, y_{2}\right)=\int_{\mathbb{R}} G\left(y_{1}^{\prime}, y_{2}\right) \mathcal{V}\left(\tau_{-y_{1}^{\prime}}(\omega), 0\right) d y_{1}^{\prime}
$$

Thus, $U_{l}$ and $\tilde{U}_{l}$ are both stationary with respect to $y_{1}$. Moreover, they are both smooth solutions of the following Stokes problem in a half space:

$$
\left\{\begin{array}{l}
-\Delta U+\nabla p=0, \quad y \in \mathbb{R}_{+}^{2} \\
\operatorname{div} U=0, \quad y \in \mathbb{R}_{+}^{2} \\
\left.U\right|_{y_{2}=0}=\left.U_{l}\right|_{y_{2}=0}
\end{array}\right.
$$

For more on the double Stokes layer potential, we refer to [13. A formal energy estimate on $U_{l}-\tilde{U}_{l}$ yields, using stationarity:

$$
\mathbb{E}\left(\int_{(-R, R) \times \mathbb{R}^{+}}\left|\nabla\left(U_{l}-\tilde{U}_{l}\right)(\cdot, y)\right|^{2} d y\right)=0, \quad \forall R>0 .
$$

One checks easily that $\nabla U_{l}$ and $\nabla \tilde{U}_{l}$ have appropriate integrability, so that this estimate is rigorous. We deduce that $U_{l}=\tilde{U}_{l}$ almost surely.

We now introduce

$$
U_{l}^{\infty}(\omega)=\lim _{R \rightarrow+\infty} \frac{1}{R} \int_{0}^{R} U_{l}\left(\omega,-y_{1}^{\prime}, 0\right) d y_{1}^{\prime} .
$$

This limit exists almost surely by the ergodic theorem, and the measurable function $U_{l}^{\infty}$ satisfies $U_{l}^{\infty} \circ \tau_{y_{1}}=U_{l}^{\infty}$ for all $y_{1} \in \mathbb{R}$. More precisely, one has

$$
U_{l}^{\infty}(\omega)=\lim _{R \rightarrow \infty} \frac{1}{R} \int_{0}^{R} U_{l}\left(\omega, y_{1}-y_{1}^{\prime}, 0\right) d y_{1}^{\prime}
$$


uniformly locally in $y_{1}$. Indeed, for all $\left|y_{1}\right|<M, R$ large enough,

$$
\begin{aligned}
& \left|\frac{1}{R} \int_{0}^{R}\left(U_{l}\left(\omega, y_{1}-y_{1}^{\prime}, 0\right)-U_{l}\left(\omega,-y_{1}^{\prime}, 0\right)\right) d y_{1}^{\prime}\right| \\
& \leq \frac{1}{R} \int_{-M}^{M} \mid U_{l}\left(\omega,-y_{1}^{\prime}, 0\left|d y_{1}^{\prime}+\frac{1}{R} \int_{R-M}^{R+M}\right| U_{l}\left(\omega,-y_{1}^{\prime}, 0 \mid d y_{1}^{\prime}\right.\right. \\
& \leq C(M) \frac{1}{2 R}\left(\int_{(-2 R, 2 R)} \mid U_{l}\left(\omega,-y_{1}^{\prime},\left.0\right|^{2} d y_{1}^{\prime}\right)^{1 / 2}\right. \\
& \leq C(M) \frac{1}{\sqrt{R}}\left(\sup _{R \geq 1} \frac{1}{R} \int_{(-R, R)} \mid U_{l}\left(\omega,-y_{1}^{\prime},\left.0\right|^{2} d y_{1}^{\prime}\right)^{1 / 2}\right.
\end{aligned}
$$

this last quantity vanishing uniformly as $R \rightarrow+\infty$, almost surely. Then, to show that $U_{l, 2}^{\infty}=0$, we integrate div $U_{l}=0$ over $\mathcal{R}_{l}^{-}(\omega, R) \cap\left\{y_{1}<0\right\}$ :

$$
0=\int_{\mathcal{R}_{l}^{-}(\omega, R) \cap\left\{y_{1}<0\right\}} \operatorname{div} U_{l}=\int_{\partial\left(\mathcal{R}_{l}^{-}(\omega, R) \cap\left\{y_{1}<0\right\}\right)} U_{l} \cdot n,
$$

which gives, as $R \rightarrow+\infty$,

$$
U_{l, 2}^{\infty}=\lim _{R \rightarrow+\infty} \frac{1}{R}\left(\int_{\sigma(-R) \cap\left\{y_{2}<0\right\}} U_{l, 1} d y_{2}-\int_{\sigma(0) \cap\left\{y_{2}<0\right\}} U_{l, 1} d y_{2}\right) .
$$

If $U_{l, 2}^{\infty}$ were not zero, one would get from the left hand side that

$$
\int_{\mathcal{R}(\omega, R)}\left|\nabla U_{l}\right|^{2} \geq C \int_{\mathcal{R}^{-}(\omega, R)}\left|U_{l}\right|^{2} \geq C \int_{R_{0}}^{R} d r\left(\int_{\sigma(-r) \cap\left\{y_{2}<0\right\}}\left|U_{l, 1}\right|+\int_{\sigma(r) \cap\left\{y_{2}<0\right\}}\left|U_{l, 1}\right|\right)^{2} \geq \delta R^{3}
$$

for some $\delta>0$ and $R \geq R_{0}$ large enough. This would be in contradiction with (2.8) b, so that $U_{l, 2}^{\infty}=0$.

We now show that almost surely,

$$
U_{l}^{\infty}(\omega)=\lim _{R \rightarrow+\infty} \frac{1}{R} \int_{0}^{R} U_{l}\left(\omega, y_{1}^{\prime}, 0\right) d y_{1}^{\prime} .
$$

Let $\tilde{U}_{l}^{\infty}$ denote this limit, which exists almost surely for the same reason as $U_{l}^{\infty}$. The convergence also holds in $L^{2}(P)$ because $\int_{0}^{1} U_{l}\left(\omega, y_{1}^{\prime}, 0\right) d y_{1}^{\prime} \in L^{2}(P)$ :

$$
\begin{aligned}
& \lim _{R \rightarrow+\infty} \mathbb{E}\left[\left|\frac{1}{R} \int_{0}^{R} U_{l}\left(\omega, y_{1}, 0\right) d y_{1}-\tilde{U}_{l}^{\infty}(\omega)\right|^{2}\right]=0, \\
& \lim _{R \rightarrow+\infty} \mathbb{E}\left[\left|\frac{1}{R} \int_{-R}^{0} U_{l}\left(\omega, y_{1}, 0\right) d y_{1}-U_{l}^{\infty}(\omega)\right|^{2}\right]=0 .
\end{aligned}
$$

In particular, we have

$$
\begin{aligned}
\mathbb{E}\left[\left|\tilde{U}_{l}^{\infty}(\omega)\right|^{2}\right] & =\lim _{R \rightarrow+\infty} \mathbb{E}\left[\left|\frac{1}{R} \int_{0}^{R} U_{l}\left(\omega, y_{1}, 0\right) d y_{1}\right|^{2}\right] \\
& =\lim _{R \rightarrow+\infty} \mathbb{E}\left[\left|\frac{1}{R} \int_{-R}^{0} U_{l}\left(\omega, y_{1}, 0\right) d y_{1}\right|^{2}\right]=\mathbb{E}\left[\left|U_{l}^{\infty}(\omega)\right|^{2}\right]
\end{aligned}
$$


since the law of $U_{l}$ is invariant by translation in the $y_{1}$-direction. Now, we write

$$
\begin{aligned}
\mathbb{E}\left[\left|\tilde{U}_{l}^{\infty}(\omega)\right|^{2}\right]= & \lim _{R \rightarrow+\infty} \mathbb{E}\left[\left|\frac{1}{2 R} \int_{0}^{2 R} U_{l}\left(\omega, y_{1}, 0\right) d y_{1}\right|^{2}\right] \\
= & \lim _{R \rightarrow+\infty}\left(\mathbb{E}\left[\left|\frac{1}{2 R} \int_{0}^{R} U_{l}\left(\omega, y_{1}, 0\right) d y_{1}\right|^{2}\right]+\mathbb{E}\left[\left|\frac{1}{2 R} \int_{R}^{2 R} U_{l}\left(\omega, y_{1}, 0\right) d y_{1}\right|^{2}\right]\right. \\
& \left.+\mathbb{E}\left[\frac{1}{2 R^{2}} \int_{0}^{R} U_{l}\left(\omega, y_{1}, 0\right) d y_{1} \int_{R}^{2 R} U_{l}\left(\omega, y_{1}, 0\right) d y_{1}\right]\right) .
\end{aligned}
$$

In this expression, the first two terms tend to the same limit $\frac{1}{4} \mathbb{E}\left[\left|\tilde{U}_{l}^{\infty}(\omega)\right|^{2}\right]$. Hence we deduce that the third term converges to $\frac{1}{2} \mathbb{E}\left[\left|\tilde{U}_{l}^{\infty}(\omega)\right|^{2}\right]$. Then we have

$$
\begin{aligned}
\mathbb{E}\left[\left|\tilde{U}_{l}^{\infty}-U_{l}^{\infty}\right|^{2}\right]= & \mathbb{E}\left[\left|\tilde{U}_{l}^{\infty}\right|^{2}\right]+\mathbb{E}\left[\left|U_{l}^{\infty}\right|^{2}\right] \\
& -2 \lim _{R \rightarrow+\infty} \mathbb{E}\left[\frac{1}{R^{2}} \int_{0}^{R} U_{l}\left(\omega, y_{1}, 0\right) d y_{1} \int_{-R}^{0} U_{l}\left(\omega, y_{1}, 0\right) d y_{1}\right] \\
= & 2 \mathbb{E}\left[\left|\tilde{U}_{l}^{\infty}\right|^{2}\right]-2 \lim _{R \rightarrow+\infty} \mathbb{E}\left[\frac{1}{R^{2}} \int_{R}^{2 R} U_{l}\left(\omega, y_{1}, 0\right) d y_{1} \int_{0}^{R} U_{l}\left(\omega, y_{1}, 0\right) d y_{1}\right]=0 .
\end{aligned}
$$

To obtain the convergence of $U_{l}$ to $U_{l}^{\infty}$ as $y_{2} \rightarrow+\infty$, we write by lemma 4.11

$$
\begin{aligned}
& U_{l}\left(\omega, y_{1}, y_{2}\right)-U_{l}^{\infty}(\omega)=\int_{\mathbb{R}} G\left(y_{1}^{\prime}, y_{2}\right)\left(U_{l}\left(\omega, y_{1}-y_{1}^{\prime}, 0\right)-U_{l}^{\infty}(\omega)\right) d y_{1}^{\prime} \\
& =\int_{\mathbb{R}} G\left(y_{1}^{\prime}, y_{2}\right) \frac{\partial}{\partial y_{1}^{\prime}} \int_{0}^{y_{1}^{\prime}} U_{l}\left(\omega, y_{1}-z, 0\right)-U_{l}^{\infty}(\omega) d z d y_{1}^{\prime} \\
& =-\int_{\mathbb{R}} \partial_{1} G\left(y_{1}^{\prime}, y_{2}\right) y_{1}^{\prime}\left(\frac{1}{y_{1}^{\prime}} \int_{0}^{y_{1}^{\prime}} U_{l}\left(\omega, y_{1}-z, 0\right) d z-U_{l}^{\infty}(\omega)\right) d y_{1}^{\prime}
\end{aligned}
$$

Let $\varepsilon, M>0$. There exists $R=R(M)>0$ such that

$$
\forall\left|y_{1}\right|<M, \forall\left|y_{1}^{\prime}\right|>R, \quad\left|\frac{1}{y_{1}^{\prime}} \int_{0}^{y_{1}^{\prime}} U_{l}\left(\omega, y_{1}-z, 0\right) d z-U_{l}^{\infty}(\omega)\right| \leq \varepsilon .
$$

We deduce that

$$
\left|\int_{\left|y_{1}^{\prime}\right|>R} \partial_{1} G\left(y_{1}^{\prime}, y_{2}\right) y_{1}^{\prime}\left(\frac{1}{y_{1}^{\prime}} \int_{0}^{y_{1}^{\prime}} U_{l}\left(\omega, y_{1}-z, 0\right) d z-U_{l}^{\infty}(\omega)\right) d y_{1}^{\prime}\right| \leq C \varepsilon
$$

Moreover,

$$
\begin{aligned}
& \left|\int_{\left|y_{1}^{\prime}\right|<R} \partial_{1} G\left(y_{1}^{\prime}, y_{2}\right) y_{1}^{\prime}\left(\frac{1}{y_{1}^{\prime}} \int_{0}^{y_{1}^{\prime}} U_{l}\left(\omega, y_{1}-z, 0\right) d z-U_{l}^{\infty}(\omega)\right) d y_{1}^{\prime}\right| \\
& \leq C\left(\int_{-R-M}^{R+M}\left|U_{l}(\omega, z, 0)\right| d z+R\left|U_{l}^{\infty}(\omega)\right|\right) \int_{\left|y_{1}^{\prime}\right|<R}\left|\partial_{1} G\left(y_{1}^{\prime}, y_{2}\right)\right| d y_{1}^{\prime} \\
& \leq C(R, M) \frac{1}{y_{2}^{2}} \underset{y_{2} \rightarrow+\infty}{\longrightarrow} 0 .
\end{aligned}
$$

This proves the almost sure convergence result. The quadratic convergence is simpler and left to the reader. 


\subsection{Some more estimates}

We will also need in the sequel the following estimates.

Proposition 12 We have for all $y_{2}>0$ and all $\alpha \in \mathbb{N}^{2}$,

$$
\sup _{R>1} \frac{1}{R} \int_{-R}^{R}\left|\partial_{y}^{\alpha} U_{l}\left(\omega, y_{1}, y_{2}\right)\right|^{2} d y_{1}<\infty \quad \text { a.s. }
$$

and for $|\alpha| \geq 1$,

$$
\sup _{R>1} \frac{y_{2}^{2|\alpha|}}{R} \mathbb{E} \int_{-R}^{R}\left|\partial_{y}^{\alpha} U_{l}\left(\cdot, y_{1}, y_{2}\right)\right|^{2} d y_{1} \underset{y_{2} \rightarrow+\infty}{\longrightarrow} 0 .
$$

Note that we could also prove an almost sure version of (4.14), as in the previous proof, but we do not need it. For $\alpha=0$, (4.14) holds if one replaces $U_{l}$ by $U_{l}-U_{l}^{\infty}$, as we have seen before.

Proof.

We have

$$
\partial^{\alpha} U_{l}\left(\omega, y_{1}, y_{2}\right)=\int \partial^{\alpha} G\left(y_{1}-y_{1}^{\prime}, y_{2}\right) U_{l}\left(\omega, y_{1}^{\prime}, 0\right) d y_{1}^{\prime}
$$

hence we have $\mathbb{E}\left[\left|\partial^{\alpha} U_{l}\left(\cdot, 0, y_{2}\right)\right|\right]<\infty$. The law of $\partial^{\alpha} U_{l}\left(\cdot, y_{1}, y_{2}\right)$ is independent of $y_{1}$. Thus we can apply the ergodic theorem, which yields (4.13). We now prove (4.14). We have

$$
\begin{aligned}
\partial^{\alpha} U_{l}\left(\omega, y_{1}, y_{2}\right) & =\int \partial^{\alpha} G\left(y_{1}^{\prime}, y_{2}\right)\left(U_{l}\left(\omega, y_{1}-y_{1}^{\prime}, 0\right)-U_{l}^{\infty}(\omega)\right) d y_{1}^{\prime} \\
& =\int y_{1}^{\prime} \partial_{1} \partial^{\alpha} G\left(y_{1}^{\prime}, y_{2}\right)\left(\frac{1}{y_{1}^{\prime}} \int_{0}^{y_{1}^{\prime}}\left(U_{l}\left(\omega, y_{1}-z, 0\right)-U_{l}^{\infty}(\omega)\right) d z\right) d y_{1}^{\prime} .
\end{aligned}
$$

Let $M>0$. We cut the previous integral in two pieces: $\left|y_{1}^{\prime}\right|<M,\left|y_{1}^{\prime}\right|>M$, and apply the Cauchy-Schwarz inequality in each piece. Recalling that $G$ is homogeneous of degree -1 , we obtain:

$$
\begin{aligned}
\left|\partial^{\alpha} U_{l}\left(y_{1}, y_{2}\right)\right|^{2} \leq & \frac{C}{y_{2}^{1+|\alpha|}} \int_{\left|y_{1}^{\prime}\right|<M}\left|\partial_{1} \partial^{\alpha} G\left(y_{1}^{\prime}, y_{2}\right)\right|\left|\int_{0}^{y_{1}^{\prime}}\left(U_{l}\left(\omega, y_{1}-z, 0\right)-U_{l}^{\infty}(\omega)\right) d z\right|^{2} d y_{1}^{\prime} \\
& +\frac{C}{y_{2}^{|\alpha|}} \int_{\left|y_{1}^{\prime}\right|>M}\left|y_{1}^{\prime} \partial_{1} \partial^{\alpha} G\left(y_{1}^{\prime}, y_{2}\right)\right|\left|\frac{1}{y_{1}^{\prime}} \int_{0}^{y_{1}^{\prime}}\left(U_{l}\left(\omega, y_{1}-z, 0\right)-U_{l}^{\infty}(\omega)\right) d z\right|^{2} d y_{1}^{\prime}
\end{aligned}
$$

We now take the expectation, and obtain, due to the invariance of the probability measure $P$ with respect to $\tau_{y_{1}}$,

$$
\begin{aligned}
\mathbb{E}\left[\left|\partial^{\alpha} U_{l}\left(\cdot, y_{1}, y_{2}\right)\right|^{2}\right] \leq & \frac{C}{y_{2}^{1+|\alpha|}} \int_{\left|y_{1}^{\prime}\right|<M}\left|\partial_{1} \partial^{\alpha} G\left(y_{1}^{\prime}, y_{2}\right)\right| \mathbb{E}\left|\int_{0}^{y_{1}^{\prime}}\left(U_{l}(\cdot,-z, 0)-U_{l}^{\infty}\right) d z\right|^{2} d y_{1}^{\prime} \\
& +\frac{C}{y_{2}^{|\alpha|}} \int_{\left|y_{1}^{\prime}\right|>M}\left|y_{1}^{\prime} \partial_{1} \partial^{\alpha} G\left(y_{1}^{\prime}, y_{2}\right)\right| \mathbb{E}\left|\frac{1}{y_{1}^{\prime}} \int_{0}^{y_{1}^{\prime}}\left(U_{l}(\cdot,-z, 0)-U_{l}^{\infty}\right) d z\right|^{2} d y_{1}^{\prime} .
\end{aligned}
$$


Hence the quantity in the l.h.s. is independent of $y_{1}$. Thus we have

$$
\begin{aligned}
\sup _{R} \frac{1}{R} \mathbb{E} \int_{-R}^{R}\left|\partial^{\alpha} U_{l}\left(\omega, y_{1}, y_{2}\right)\right|^{2} d y_{1} \leq & \frac{C}{y_{2}^{2+2|\alpha|}} \mathbb{E}\left|\int_{-M}^{M}\left(U_{l}(\omega,-z, 0)-U_{l}^{\infty}\right) d z\right|^{2} \\
& +\frac{C}{y_{2}^{2|\alpha|}} \sup _{\left|y_{1}^{\prime}\right|>M} \mathbb{E}\left|\frac{1}{y_{1}^{\prime}} \int_{0}^{y_{1}^{\prime}}\left(U_{l}(\omega,-z, 0)-U_{l}^{\infty}\right) d z\right|^{2} \\
\leq & \frac{C}{y_{2}^{2+2|\alpha|}}+\frac{C \delta}{y_{2}^{2|\alpha|}}
\end{aligned}
$$

provided $M$ is chosen great enough so that $\mathbb{E}\left|\frac{1}{y_{1}^{\prime}} \int_{0}^{y_{1}^{\prime}}\left(U_{l}(\cdot,-z, 0)-U_{l}^{\infty}\right) d z\right|^{2}<\delta$ for all $\left|y_{1}^{\prime}\right|>$ $M$. The result follows.

\section{$5 \quad$ Justification of Navier's law}

This section is divided into two parts. First we derive an approximation $\tilde{u}_{a p p}^{\varepsilon}$ of $u^{\varepsilon}$ based on the boundary layer analysis, and prove theorem 4 . Then using this approximation, we justify Navier's wall law and prove theorem 5 .

\subsection{Approximation of $u^{\varepsilon}$}

The approximation $\tilde{u}_{a p p}^{\varepsilon}$ reads

$$
\begin{aligned}
\tilde{u}_{\text {app }}^{\varepsilon}(\omega, x)= & u^{0}(\omega, x)+\varepsilon U_{l}\left(\omega, \frac{x_{1}}{\varepsilon}, \frac{x_{2}}{\varepsilon}\right)+\varepsilon U_{u}\left(\omega, \frac{x_{1}}{\varepsilon}, \frac{x_{2}-1}{\varepsilon}\right) \\
& +\varepsilon u^{1, \varepsilon}(\omega, x)
\end{aligned}
$$

It takes into account the boundary layer terms, and includes corrections to recover the correct boundary and flux conditions : this is the role of $u^{1, \varepsilon}$.

More precisely, since $U_{l, u}$ does not converges to 0 when $y_{2}$ tends to $\pm \infty$, we subtract $U_{l, u}^{\infty}$ from $U_{l, u}$ in (2.6). Then we deal with the boundary condition. The term $\varepsilon\left(U_{l}-U_{l}^{\infty}\right)$ is non zero on the upper boundary of $\Omega^{\varepsilon}$. We truncate this term replacing it by $\varepsilon\left(U_{l}-U_{l}^{\infty}\right) 1_{x_{2}<1}$. Hence we have the correct boundary condition, but a jump appears on $\Sigma_{1}$. So we introduce a function $\varepsilon v_{l}(x)$ such that $v_{l}\left(x_{1}, 1\right)=U_{l}^{\infty}-U_{l}\left(\frac{x_{1}}{\varepsilon}, \frac{1}{\varepsilon}\right)$ on $\Sigma_{1}, v_{l}=0$ on $\Sigma_{0}$, with div $v_{l}=0$ in $\Omega$, and we set $v_{l}=0$ outside $\Omega$. Notice that $\varepsilon\left(U_{l}-U_{l}^{\infty}\right)=o(\varepsilon)$, thus $\varepsilon v_{l}=o(\varepsilon)$ (roughly), so this term will not interfere in our estimate.

Now on the lower boundary, we have $\varepsilon\left(U_{l}-U_{l}^{\infty}\right)=-\varepsilon U_{l}^{\infty}$. So we will again introduce a counterflow $\varepsilon c_{l}$ in $\Omega$. Unlike $\varepsilon v_{l}, \varepsilon c_{l}$ is not $o(\varepsilon)$, thus we have to choose for $c_{l}$ a solution of the following equations in $\Omega$ :

$$
\left\{\begin{array}{l}
-\nu \Delta c_{l}+\left(u^{0} \cdot \nabla\right) c_{l}+\left(c_{l} \cdot \nabla\right) u^{0}+\nabla g_{l}=0 \\
\operatorname{div} c_{l}=0 \\
\left.c_{l}\right|_{\Sigma_{0}}=U_{l, 1}^{\infty} e_{1},\left.\quad c_{l}\right|_{\Sigma_{1}}=0 \\
\int_{0}^{1} c_{l} \cdot e_{1} d x_{2}=0
\end{array}\right.
$$


(recall that $U_{l, 2}^{\infty}=0$ ). The solution of this system is just a combination of a Couette flow and a Poiseuille flow: $c_{l}=\left(1-4 x_{2}+3 x_{2}^{2}\right) U_{l, 1}^{\infty} e_{1}, \nabla g_{l}=6 \nu U_{l}^{\infty}$. We extend $c_{l}$ outside $\Omega$ by setting $c_{l}=0$ above $\Sigma_{1}$ and $c_{l}=U_{l}^{\infty}$ below $\Sigma_{0}$.

We proceed similarly with $\varepsilon\left(U_{u}-U_{u}^{\infty}\right)$, introducing $v_{u}$ and $c_{u}$. We have $c_{u}=\left(3 x_{2}^{2}-2 x_{2}\right) U_{u}^{\infty}$ in $\Omega, c_{u}=U_{u}^{\infty}$ above $\Sigma_{1}$ and $c_{u}=0$ below $\Sigma_{0}$. Finally, we add a small Poiseuille flow $\varepsilon \theta u_{0}$ to correct the value of the flux of $u_{a p p}^{\varepsilon}$. We obtain:

$$
\tilde{u}_{a p p}^{\varepsilon}=u^{0}+\varepsilon\left(U_{l}\left(\frac{x}{\varepsilon}\right)-U_{l}^{\infty}\right) 1_{x_{2}<1}+\varepsilon\left(U_{u}\left(\frac{x}{\varepsilon}\right)-U_{u}^{\infty}\right) 1_{x_{2}>0}+\varepsilon\left(v_{l}+v_{u}+c_{l}+c_{u}\right)+\varepsilon \theta u^{0}
$$

where the coefficient $\theta$ is defined by

$$
\theta \phi=-\int_{\sigma\left(x_{1}\right)}\left(U_{l, 1}\left(\frac{x}{\varepsilon}\right) 1_{x_{2}<1}+U_{u, 1}\left(\frac{x}{\varepsilon}\right) 1_{x_{2}>0}\right) d x_{2}+U_{l, 1}^{\infty}+U_{u, 1}^{\infty}-\int_{0}^{1}\left(v_{l, 1}(x)+v_{u, 1}(x)\right) d x_{2} .
$$

The value of $\theta$ is independent of $x_{1}$, because $\operatorname{div} \tilde{u}_{a p p}^{\varepsilon}=0$. Easy recombinations show that the expansion (5.2) is of type (5.1), with

$$
u^{1, \varepsilon}=u^{1}+v_{l}+v_{u}+\theta u^{0}-\left(U_{l}\left(\frac{x}{\varepsilon}\right)-U_{l}^{\infty}\right) 1_{x_{2}>1}+\varepsilon\left(U_{u}\left(\frac{x}{\varepsilon}\right)-U_{u}^{\infty}\right) 1_{x_{2}<0},
$$

and $u^{1}=c_{l}+c_{u}-U_{l}^{\infty}-U_{u}^{\infty}$ (according to (4.1) and (4.2)).

\subsubsection{Construction of $v_{l}$ and $v_{u}$}

We explain in this paragraph the construction of the flow $v_{l}$. The construction of $v_{u}$ is analogous. We have to solve the following problem: find $v_{l} \in H_{l o c}^{1}(\Omega)$ such that

$$
\left\{\begin{array}{l}
v_{l}\left(x_{1}, 0\right)=0 \text { on } \Sigma_{0} \\
v_{l}\left(x_{1}, 1\right)=U_{l}^{\infty}-U_{l}\left(\frac{x_{1}}{\varepsilon}, \frac{1}{\varepsilon}\right) \text { on } \Sigma_{1} \\
\operatorname{div} v_{l}=0 \text { in } \Omega .
\end{array}\right.
$$

Proposition 13 This problem possesses a (non unique) solution $v_{l}$ such that

$$
\sup _{R>1} \frac{1}{R}\left\|v_{l}\right\|_{H^{2}\left(\Omega_{R}\right)}^{2}<\infty \quad \text { a.s. }
$$

and

$$
\sup _{R>1} \frac{1}{R} \mathbb{E}\left(\left\|v_{l}\right\|_{H^{2}\left(\Omega_{R}\right)}^{2}\right)=o(1)
$$

when $\varepsilon \rightarrow 0$.

Proof.

We shall find a solution in the following form: $v_{l}=\nabla^{\perp} \psi$. In terms of $\psi$, the boundary conditions can be rewritten as $\partial_{1} \psi=\partial_{2} \psi=0$ on $\Sigma_{0}, \partial_{1} \psi\left(x_{1}, 1\right)=-U_{l, 2}\left(\frac{x_{1}}{\varepsilon}, \frac{1}{\varepsilon}\right)$ and $\partial_{2} \psi\left(x_{1}, 1\right)=U_{l, 1}\left(\frac{x_{1}}{\varepsilon}, \frac{1}{\varepsilon}\right)-U_{l, 1}^{\infty}$ on $\Sigma_{1}$. Up to a constant, this is equivalent to $\psi=\partial_{2} \psi=0$ on $\Sigma_{0}, \psi\left(x_{1}, 1\right)=-\int_{0}^{x_{1}} U_{l, 2}\left(\frac{x_{1}^{\prime}}{\varepsilon}, \frac{1}{\varepsilon}\right) d x_{1}^{\prime}, \partial_{2} \psi\left(x_{1}, 1\right)=U_{l, 1}\left(\frac{x_{1}}{\varepsilon}, \frac{1}{\varepsilon}\right)-U_{l, 1}^{\infty}$ on $\Sigma_{1}$. We search a 
solution which is polynomial in $x_{2}$ : let $\psi\left(x_{1}, x_{2}\right)=a\left(x_{1}\right) x_{2}^{3}+b\left(x_{1}\right) x_{2}^{2}+c\left(x_{1}\right) x_{2}+d\left(x_{1}\right)$. The boundary conditions on $\Sigma_{0}$ imply $c\left(x_{1}\right)=d\left(x_{1}\right)=0$, and on $\Sigma_{1}$ we get

$$
\left\{\begin{aligned}
a+b & =-\int_{0}^{x_{1}} U_{l, 2}\left(\frac{x_{1}^{\prime}}{\varepsilon}, \frac{1}{\varepsilon}\right) d x_{1}^{\prime} \\
3 a+2 b & =U_{l, 1}\left(\frac{x_{1}}{\varepsilon}, \frac{1}{\varepsilon}\right)-U_{l, 1}^{\infty} .
\end{aligned}\right.
$$

This system has of course a unique solution $\left(a\left(x_{1}\right), b\left(x_{1}\right)\right)$, and to obtain the required estimates, it is sufficient to show that

$$
\begin{aligned}
& \sup _{R>1} \frac{1}{R}\left(\left\|\int_{0}^{x_{1}} U_{l, 2}\left(\frac{x_{1}^{\prime}}{\varepsilon}, \frac{1}{\varepsilon}\right) d x_{1}^{\prime}\right\|_{H^{3}(-R, R)}^{2}+\left\|U_{l, 1}\left(\frac{x_{1}}{\varepsilon}, \frac{1}{\varepsilon}\right)-U_{l, 1}^{\infty}\right\|_{H^{3}(-R, R)}^{2}\right)<\infty \quad \text { a.s. } \\
& \quad \sup _{R>1} \frac{1}{R} \mathbb{E}\left[\left\|\int_{0}^{x_{1}} U_{l, 2}\left(\frac{x_{1}^{\prime}}{\varepsilon}, \frac{1}{\varepsilon}\right) d x_{1}^{\prime}\right\|_{H^{3}(-R, R)}^{2}+\left\|U_{l, 1}\left(\frac{x_{1}}{\varepsilon}, \frac{1}{\varepsilon}\right)-U_{l, 1}^{\infty}\right\|_{H^{3}(-R, R)}^{2}\right] \rightarrow 0 .
\end{aligned}
$$

The estimate on the quantity $\left\|U_{l}\left(\frac{x_{1}}{\varepsilon}, \frac{1}{\varepsilon}\right)-U_{l}^{\infty}\right\|_{H^{3}(-R, R)}^{2}$ follows from proposition 12, for we have

$$
\begin{aligned}
\frac{1}{R}\left\|U_{l}\left(\frac{x_{1}}{\varepsilon}, \frac{1}{\varepsilon}\right)-U_{l}^{\infty}\right\|_{H^{3}(-R, R)}^{2}=\frac{\varepsilon}{R} \int_{-R / \varepsilon}^{R / \varepsilon}( & \left(U_{l}\left(y_{1}, \frac{1}{\varepsilon}\right)-\left.U_{l}^{\infty}\right|^{2}+\varepsilon^{-2}\left|\partial_{1} U_{l}\left(y_{1}, \frac{1}{\varepsilon}\right)\right|^{2}\right. \\
& \left.+\varepsilon^{-4}\left|\partial_{1}^{2} U_{l}\left(y_{1}, \frac{1}{\varepsilon}\right)\right|^{2}+\varepsilon^{-6}\left|\partial_{1}^{3} U_{l}\left(y_{1}, \frac{1}{\varepsilon}\right)\right|^{2}\right) d y_{1}
\end{aligned}
$$

(recall that $U_{l, 2}^{\infty}=0$ ). Hence we only need to deal with $\frac{1}{R}\left\|\int_{0}^{x_{1}} U_{l, 2}\left(\frac{x_{1}^{\prime}}{\varepsilon}, \frac{1}{\varepsilon}\right) d x_{1}^{\prime}\right\|_{L^{2}(-R, R)}^{2}$. In order to do so, we write:

$$
\begin{aligned}
\int_{0}^{x_{1}} U_{l, 2}\left(\frac{x_{1}^{\prime}}{\varepsilon}, \frac{1}{\varepsilon}\right) d x_{1}^{\prime} & =\varepsilon \int_{0}^{x_{1} / \varepsilon} U_{l, 2}\left(y_{1}, \frac{1}{\varepsilon}\right) d y_{1} \\
& =\varepsilon \int_{\sigma\left(-\frac{x_{1}}{\varepsilon}\right) \cap\left\{y_{2}<\frac{1}{\varepsilon}\right\}} U_{l, 1}\left(\frac{x_{1}}{\varepsilon}, y_{2}\right) d y_{2}-\varepsilon \int_{\sigma(0) \cap\left\{y_{2}<\frac{1}{\varepsilon}\right\}} U_{l, 1}\left(0, y_{2}\right) d y_{2}
\end{aligned}
$$

since $U_{l}$ is divergence free on $\mathcal{R}_{l} \cap\left\{0<y_{1}<\frac{x_{1}}{\varepsilon}, y_{2}<\frac{1}{\varepsilon}\right\}$. Now the pointwise estimate is easy, for we have

$$
\mathbb{E}\left|\varepsilon \int_{\sigma(0) \cap\left\{y_{2}<\frac{1}{\varepsilon}\right\}} U_{l, 1}\left(0, y_{2}\right) d y_{2}\right|^{2}<\infty
$$

hence we can apply the pointwise ergodic theorem. Next we turn to the estimate in expectation. We have

$$
\begin{aligned}
\int_{0}^{x_{1}} U_{l, 2}\left(\frac{x_{1}^{\prime}}{\varepsilon}, \frac{1}{\varepsilon}\right) d x_{1}^{\prime}= & \varepsilon \int_{0}^{1 / \varepsilon}\left(U_{l, 1}\left(\frac{x_{1}}{\varepsilon}, y_{2}\right)-U_{l, 1}^{\infty}\right) d y_{2}-\varepsilon \int_{0}^{1 / \varepsilon}\left(U_{l, 1}\left(0, y_{2}\right)-U_{l, 1}^{\infty}\right) d y_{2} \\
& +\varepsilon \int_{\sigma\left(-\frac{x_{1}}{\varepsilon}\right) \cap\left\{y_{2}<0\right\}} U_{l, 1}\left(\frac{x_{1}}{\varepsilon}, y_{2}\right) d y_{2}-\varepsilon \int_{\sigma(0) \cap\left\{y_{2}<0\right\}} U_{l, 1}\left(0, y_{2}\right) d y_{2}
\end{aligned}
$$

Then the invariance of $P$ yields

$$
\mathbb{E} \frac{1}{2 R}\left\|\varepsilon \int_{0}^{1 / \varepsilon}\left(U_{l, 1}\left(\frac{x_{1}}{\varepsilon}, y_{2}\right)-U_{l, 1}^{\infty}\right) d y_{2}\right\|_{L^{2}(-R, R)}^{2}=\mathbb{E}\left|\varepsilon \int_{0}^{1 / \varepsilon}\left(U_{l, 1}\left(0, y_{2}\right)-U_{l, 1}^{\infty}\right) d y_{2}\right|^{2} .
$$


Therefore it is sufficient to deal with the integral of the l.h.s. with $R=1$. Let $\delta>0$. We have

$$
\begin{aligned}
\mathbb{E}\left\|\varepsilon \int_{0}^{1 / \varepsilon}\left(U_{l, 1}\left(\frac{x_{1}}{\varepsilon}, y_{2}\right)-U_{l, 1}^{\infty}\right) d y_{2}\right\|_{L^{2}(-1,1)}^{2} & \leq \varepsilon \int_{0}^{1 / \varepsilon} \mathbb{E}\left[\int_{-1}^{1}\left|U_{l, 1}\left(\frac{x_{1}}{\varepsilon}, y_{2}\right)-U_{l, 1}^{\infty}\right|^{2} d x_{1}\right] d y_{2} \\
& \leq \varepsilon \int_{0}^{1 / \varepsilon} \mathbb{E}\left[\int_{-1}^{1}\left|U_{l, 1}\left(y_{1}, y_{2}\right)-U_{l, 1}^{\infty}\right|^{2} d y_{1}\right] d y_{2} \\
& \leq \varepsilon \mathbb{E} \int_{[-1,1] \times[0, M]}\left|U_{l, 1}-U_{l, 1}^{\infty}\right|^{2}+\varepsilon \int_{M}^{1 / \varepsilon} \delta d y_{2} \\
& \leq C \varepsilon+\delta,
\end{aligned}
$$

where we have used successively the Cauchy-Schwarz inequality, the invariance of $P$ and the estimate (2.9) to make the expectation of $\int_{-1}^{1}\left|U_{l, 1}-U_{l, 1}^{\infty}\right|^{2} d y_{1}$ less than $\delta$ if $y_{2}$ is larger than some constant $M$. Letting $\varepsilon$ decrease to 0 , we deduce that the l.h.s. above tends to 0 . It remains to deal with the integrals on $\sigma\left(\frac{x_{1}}{\varepsilon}\right) \cap\left\{y_{2}<0\right\}$ and $\sigma(0) \cap\left\{y_{2}<0\right\}$. As above, the expectations of the quadratic mean on $(-R, R)$ of these two integrals are equal, and equal to:

$$
\begin{aligned}
\mathbb{E} \frac{1}{2 R}\left\|\varepsilon \int_{\sigma\left(-\frac{x_{1}}{\varepsilon}\right) \cap\left\{y_{2}<0\right\}} U_{l, 1}\left(\frac{x_{1}}{\varepsilon}, y_{2}\right) d y_{2}\right\|_{L^{2}(-R, R)}^{2} & =\mathbb{E}\left|\varepsilon \int_{\sigma(0) \cap\left\{y_{2}<0\right\}} U_{l, 1}\left(0, y_{2}\right) d y_{2}\right|^{2} \\
& =\mathbb{E} \int_{0}^{1}\left|\varepsilon \int_{\sigma\left(y_{1}\right) \cap\left\{y_{2}<0\right\}} U_{l, 1}\left(y_{1}, y_{2}\right) d y_{2}\right|^{2} d y_{1} \\
& \leq \varepsilon^{2} \mathbb{E} \int_{0}^{1} \int_{\sigma\left(y_{1}\right) \cap\left\{y_{2}<0\right\}}\left|U_{l, 1}\left(y_{1}, y_{2}\right)\right|^{2} d y_{2} d y_{1} \\
& \leq C \varepsilon^{2} .
\end{aligned}
$$

Putting together the four previous estimates, we can conclude that

$$
\sup _{R} \frac{1}{R} \mathbb{E}\left\|\int_{0}^{x_{1}} U_{l, 2}\left(\frac{x_{1}^{\prime}}{\varepsilon}, \frac{1}{\varepsilon}\right) d x_{1}^{\prime}\right\|_{L^{2}(-R, R)}^{2} \rightarrow 0 .
$$

This ends the proof of proposition 13 .

\subsubsection{Proof of theorem 1}

This paragraph is devoted to the estimates (2.10). It is enough to derive the first inequality, as the second one follows directly from Poincaré inequality. In fact, we will show that

$$
\sup _{R \geq 1} \frac{1}{R} \mathbb{E}\left(\int_{\Omega^{\varepsilon}(\cdot, R)}\left|\nabla u^{\varepsilon}-\nabla \tilde{u}_{a p p}^{\varepsilon}\right|^{2}\right)=o\left(\varepsilon^{2}\right)
$$

where $\tilde{u}_{a p p}^{\varepsilon}$ is given by (5.1)-(5.2). Indeed, one verifies easily from the previous estimates that

$$
\sup _{R \geq 1} \frac{1}{R} \mathbb{E}\left(\int_{\Omega^{\varepsilon}(\cdot, R)}\left|\nabla u_{\text {app }}^{\varepsilon}-\nabla \tilde{u}_{\text {app }}^{\varepsilon}\right|^{2}\right)=o\left(\varepsilon^{2}\right) .
$$


We write $w=u^{\varepsilon}-\tilde{u}_{a p p}^{\varepsilon}=v^{\varepsilon}-v_{a p p}^{\varepsilon}, v^{\varepsilon}=u^{\varepsilon}-u^{0}, v_{a p p}^{\varepsilon}=\tilde{u}_{a p p}^{\varepsilon}-u^{0}$. It satisfies (3.2) with $v=0$,

$$
\left\{\begin{array}{l}
f(\omega, x)=0, \quad x \text { in } \Omega \\
f(\omega, x)=(-12 \nu \phi(1+\varepsilon \theta), 0), \quad x \text { in } \Omega^{\varepsilon}(\omega) \backslash \Omega
\end{array}\right.
$$

and

$$
\left.\tilde{\phi}\right|_{\Sigma_{0,1}}= \pm\left.\left[\partial_{2} U_{u, l}\left(\frac{x}{\varepsilon}\right)\right]\right|_{\Sigma_{0,1}}+\left.\varepsilon \partial_{2}\left(v_{l}+v_{u}+c_{l}+c_{u}\right)\right|_{\Sigma_{0,1}}+6 \varepsilon \theta \phi
$$

Finally, using that

$$
\operatorname{div}\left(u^{0} \otimes\left(U_{l}^{\infty}+U_{u}^{\infty}+u^{1}\right)\right)=\operatorname{div}\left(\left(U_{l}^{\infty}+U_{u}^{\infty}+u^{1}\right) \otimes u^{0}\right)=0,
$$

where $u^{1}$ is given in (4.2), we write

$$
\begin{aligned}
G= & -v^{\varepsilon} \otimes v^{\varepsilon}+u^{0} \otimes\left(v_{a p p}^{\varepsilon}-\varepsilon\left(U_{l}^{\infty}+U_{u}^{\infty}+u^{1}\right)\right) \\
& +\left(v_{a p p}^{\varepsilon}-\varepsilon\left(U_{l}^{\infty}+U_{u}^{\infty}+u^{1}\right)\right) \otimes u^{0}+\nu \varepsilon \nabla\left(v_{l}+v_{u}\right) .
\end{aligned}
$$

Proceeding as in section 3, one has

$$
\begin{aligned}
\int_{\eta}^{\eta+1} d R \int_{\Omega^{\varepsilon}(R)}|\nabla w|^{2} \leq & C\left(\int_{\eta}^{\eta+1} d R \int_{\Omega^{\varepsilon}(R)}\left(|G|^{2}+\varepsilon^{2}|f|^{2}\right)+\varepsilon \int_{\eta}^{\eta+1} d R \int_{\Sigma_{0}(R) \cup \Sigma_{1}(R)}|\tilde{\phi}|^{2}\right. \\
& \left.+\int_{\Omega^{\varepsilon}(\eta, \eta+1)}\left(|G|^{2}+\varepsilon^{2}|f|^{2}\right)+\int_{\Omega^{\varepsilon}(\eta, \eta+1)}|\nabla w|^{2}\right)
\end{aligned}
$$

which yields roughly, after integration with respect to $\omega$ :

$$
\begin{aligned}
\mathbb{E} \int_{\eta}^{\eta+1} d R\left(\int_{\Omega^{\varepsilon}(R)}|\nabla w|^{2}\right) \leq & C\left(\mathbb{E} \int_{\Omega^{\varepsilon}(\eta+1)}\left(|G|^{2}+\varepsilon^{2}|f|^{2}\right)+\varepsilon \mathbb{E} \int_{\Sigma_{0}(\eta+1) \cup \Sigma_{1}(\eta+1)}|\tilde{\phi}|^{2}\right. \\
& \left.+\mathbb{E} \int_{\Omega^{\varepsilon}(\eta, \eta+1)}|\nabla w|^{2}\right) .
\end{aligned}
$$

Assuming that

$$
\begin{aligned}
& \mathbb{E} \int_{\Omega^{\varepsilon}(\eta+1)}|G|^{2}+\varepsilon^{2}|f|^{2} \leq \delta(\varepsilon) \varepsilon^{2}(\eta+1), \quad \delta(\varepsilon) \underset{\varepsilon \rightarrow 0}{\longrightarrow} 0, \\
& \mathbb{E} \int_{\Sigma_{0,1}(\eta+1)}|\tilde{\phi}|^{2} \leq C \varepsilon^{2}(\eta+1),
\end{aligned}
$$

we end up with a reverse Gronwall inequality:

$$
F(\eta) \leq \eta(\varepsilon) \varepsilon^{2}(\eta+1)+C F^{\prime}(\eta), \quad F(\eta)=\mathbb{E} \int_{\eta}^{\eta+1} d R\left(\int_{\Omega^{\varepsilon}(R)}|\nabla w|^{2}\right), \quad \eta(\varepsilon) \underset{\varepsilon \rightarrow 0}{\longrightarrow} 0,
$$

and conclude as in section 3. It thus remains to establish bounds (5.3). The second inequality is obvious using propositions 12 and 13 . To control $G$, we first notice that

$$
\begin{aligned}
\int_{\Omega^{\varepsilon}(\eta+1)}\left|v^{\varepsilon} \otimes v^{\varepsilon}\right|^{2} & \leq C(\eta+1) \sup _{|k| \leq \eta+2}\left\|v^{\varepsilon}\right\|_{L^{4}\left(\Omega^{\varepsilon}(k, k+1)\right)}^{4} \\
& \leq C(\eta+1)\left\|v^{\varepsilon}\right\|_{L_{\text {uloc }}^{2}}^{2}\left\|\nabla v^{\varepsilon}\right\|_{L_{\text {uloc }}^{2}}^{2}
\end{aligned}
$$


by standard Gagliardo-Nirenberg inequality. Using estimates (3.19), (3.21), we deduce

$$
\int_{\Omega^{\varepsilon}(\eta+1)}\left|v^{\varepsilon} \otimes v^{\varepsilon}\right|^{2} \leq C \varepsilon^{3}(\eta+1) .
$$

Then, with notations of (5.1) we write

$$
\begin{aligned}
& v_{\text {app }}^{\varepsilon}(\omega, x)-\varepsilon\left(U_{l}^{\infty}(\omega)-U_{u}^{\infty}(\omega)-u^{1}(\omega, x)\right)=\varepsilon\left(\left(U_{l}^{\infty}\left(\omega, \frac{x}{\varepsilon}\right)-U_{l}^{\infty}(\omega)\right)\right. \\
& \left.+\varepsilon\left(U_{u}^{\infty}\left(\omega, \frac{x_{1}}{\varepsilon}, \frac{x_{2}-1}{\varepsilon}\right)-U_{u}^{\infty}(\omega)\right)+\varepsilon\left(u^{1, \varepsilon}(\omega, x)-u^{1}(\omega, x)\right)\right) .
\end{aligned}
$$

In particular, the estimate of $G$ involves

$$
\begin{aligned}
& \mathbb{E}\left(\int_{\Omega^{\varepsilon}(\omega, \eta+1)}\left|U_{u}^{\infty}\left(\omega, \frac{x_{1}}{\varepsilon}, \frac{x_{2}-1}{\varepsilon}\right)-U_{u}^{\infty}(\omega)\right| d x\right) d \omega \\
& \leq C(\eta+1) \mathbb{E}\left(\varepsilon \int_{-h_{l}(0)}^{1 / \varepsilon+h_{u}(0)}\left|U_{l}\left(\omega, 0, y_{2}\right)-U_{\infty}^{l}(\omega)\right|^{2} d y_{2}\right) d \omega=o(1), \quad \varepsilon \rightarrow 0,
\end{aligned}
$$

with the same manipulations as in the proof of proposition 13. All other terms lead to similar inequalities and are left to the reader. This ends the proof.

\subsection{Effective wall law}

It remains to connect the approximation we have built to the Navier's wall law. Following [15], such connection can be seen at a formal level, as

$$
u^{\varepsilon} \approx u_{\text {app }}^{\varepsilon}=u^{0}(\omega, x)+\varepsilon U_{l}\left(\omega, \frac{x_{1}}{\varepsilon}, \frac{x_{2}}{\varepsilon}\right)+\varepsilon U_{u}\left(\omega, \frac{x_{1}}{\varepsilon}, \frac{x_{2}-1}{\varepsilon}\right)+\varepsilon u^{1}(\omega, x),
$$

For instance, we obtain formally:

$$
\left.u_{1}^{\varepsilon}\right|_{\Sigma_{0}} \approx \varepsilon U_{l, 1}\left(\omega, \frac{x_{1}}{\varepsilon}, 0\right),\left.\quad \partial_{2} u_{1}^{\varepsilon}\right|_{\Sigma_{0}} \approx 6 \phi+\partial_{2} U_{l, 1}\left(\omega, \frac{x_{1}}{\varepsilon}, 0\right)
$$

which yields after averaging the approximate boundary condition,

$$
\left.v_{1}^{\varepsilon}\right|_{\Sigma_{0}}=\left.\varepsilon \alpha_{l} \partial_{2} v_{1}^{\varepsilon}\right|_{\Sigma_{0}} .
$$

Similarly,

$$
\left.v_{1}^{\varepsilon}\right|_{\Sigma_{1}}=-\left.\varepsilon \alpha_{u} \partial_{2} v_{1}^{\varepsilon}\right|_{\Sigma_{1}}
$$

We now justify this formal computation and prove theorem 5. Let $v^{\varepsilon}$ the solution of (2.11)-(2.12). It is given explicitly by

$$
v^{\varepsilon}=\left(M_{\phi}\left(x_{2}^{2}-\left(x_{2}-\varepsilon \alpha_{l}\right) \frac{1-2 \varepsilon \alpha_{u}}{2\left(1-\varepsilon\left(\alpha_{l}+\alpha_{u}\right)\right)}\right), 0\right), \quad \phi M_{\phi}=\frac{1}{3}-\frac{\left(1-2 \varepsilon \alpha_{l}\right)\left(1-2 \varepsilon \alpha_{u}\right)}{1-\varepsilon\left(\alpha_{l}+\alpha_{u}\right)} .
$$

Then, we use the identity:

$$
\begin{aligned}
u^{\varepsilon}(\omega, x)-v^{\varepsilon}(\omega, x)= & u^{\varepsilon}(\omega, x)-u_{\text {app }}^{\varepsilon}(\omega, x)+u_{\text {app }}^{\varepsilon}(\omega, x)-v^{\varepsilon}(\omega, x)=\left(u^{\varepsilon}-u_{\text {app }}^{\varepsilon}\right)(\omega, x) \\
& +\varepsilon\left(u^{0}(\omega, x)+\varepsilon u^{1}(\omega, x)-U_{l}^{\infty}(\omega)-U_{u}^{\infty}(\omega)\right)-v^{\varepsilon}(\omega, x) \\
& +\varepsilon\left(U_{l}\left(\omega, \frac{x_{1}}{\varepsilon}, \frac{x_{2}}{\varepsilon}\right)-U_{l}^{\infty}(\omega)\right)+\varepsilon\left(U_{u}\left(\omega, \frac{x_{1}}{\varepsilon}, \frac{x_{2}-1}{\varepsilon}\right)-U_{u}^{\infty}(\omega)\right) .
\end{aligned}
$$


By theorem 因, we have

$$
\sup _{R \geq 1} \frac{1}{R} \mathbb{E}\left(\int_{\Omega(R)}\left|u^{\varepsilon}(\omega, \cdot)-u_{\text {app }}^{\varepsilon}(\omega, \cdot)\right|^{2}\right)=o\left(\varepsilon^{2}\right) .
$$

Then, simple calculations show that the function

$$
w=\varepsilon\left(u^{0}(\omega, x)+\varepsilon u^{1}(\omega, x)-U_{l}^{\infty}(\omega)-U_{u}^{\infty}(\omega)\right)-v^{\varepsilon}(\omega, x)
$$

which is the solution of

$$
\left\{\begin{array}{l}
w \cdot \nabla w-\nu \Delta w+\nabla q=0, \quad x \in \Omega, \\
\operatorname{div} w=0, \quad x \in \Omega, \\
\int_{\sigma\left(x_{1}\right)} w_{1}=0,\left.\quad w_{2}(\omega, \cdot)\right|_{\Sigma_{0,1}}=0, \\
w(\omega, \cdot)-\varepsilon \alpha_{l}(\omega) \frac{\partial w(\omega, \cdot)}{\partial x_{2}}=\varepsilon^{2} \alpha_{l}(\omega)\left(4 U_{l, 1}^{\infty}(\omega)+2 U_{u, 1}^{\infty}(\omega)\right), \quad x_{2}=0, \\
w(\omega, \cdot)+\varepsilon \alpha_{u}(\omega) \frac{\partial w(\omega, \cdot)}{\partial x_{2}}=\varepsilon^{2} \alpha_{u}(\omega)\left(2 U_{l, 1}^{\infty}(\omega)+4 U_{u, 1}^{\infty}(\omega)\right), \quad x_{2}=1,
\end{array}\right.
$$

satisfies

$$
\sup _{R \geq 1} \frac{1}{R} \mathbb{E}\left(\int_{\Omega(R)}\left|u^{\varepsilon}(\omega, \cdot)-u_{\text {app }}^{\varepsilon}(\omega, \cdot)\right|^{2}\right)=O\left(\varepsilon^{4}\right) .
$$

Finally, we proceed as usual to get

$$
\sup _{R \geq 1} \frac{1}{R} \mathbb{E}\left(\int_{\Omega(R)}\left|U_{l}\left(\omega, \frac{x_{1}}{\varepsilon}, \frac{x_{2}}{\varepsilon}\right)-U_{l}^{\infty}(\omega)\right|^{2} d x\right) d \omega=o(1),
$$

and similar estimate for the upper boundary layer. This concludes the proof of theorem 5 .

\section{References}

[1] Y. Abddaimi and G. Michaille. Dirichlet problem associated with a random quasilinear operator in a random domain. RAIRO Modél. Math. Anal. Numér., 30(1):103-121, 1996.

[2] Y. Achdou, P. Le Tallec, F. Valentin, and O. Pironneau. Constructing wall laws with domain decomposition or asymptotic expansion techniques. Comput. Methods Appl. Mech. Engrg., 151(1-2):215-232, 1998. Symposium on Advances in Computational Mechanics, Vol. 3 (Austin, TX, 1997).

[3] Yves Achdou, O. Pironneau, and F. Valentin. Effective boundary conditions for laminar flows over periodic rough boundaries. J. Comput. Phys., 147(1):187-218, 1998.

[4] Yves Achdou and Olivier Pironneau. Domain decomposition and wall laws. C. R. Acad. Sci. Paris Sér. I Math., 320(5):541-547, 1995.

[5] Youcef Amirat, Didier Bresch, Jérôme Lemoine, and Jacques Simon. Effect of rugosity on a flow governed by stationary Navier-Stokes equations. Quart. Appl. Math., 59(4):769$785,2001$. 
[6] D.W. Bechert and M. Bartenwerfer. The viscous flow on surfaces with longitudinal ribs. J. Fluid Mech., 206(01):105-129, 1989.

[7] A. Yu. Beliaev and S. M. Kozlov. Darcy equation for random porous media. Comm. Pure Appl. Math., 49(1):1-34, 1996.

[8] Alain Bourgeat, Andro Mikelić, and Steve Wright. Stochastic two-scale convergence in the mean and applications. J. Reine Angew. Math., 456:19-51, 1994.

[9] Didier Bresch and David Gérard-Varet. Roughness-induced effects on the quasigeostrophic model. Comm. Math. Phys., 253(1):81-119, 2005.

[10] C. Castaing and M. Valadier. Convex analysis and measurable multifunctions. SpringerVerlag, Berlin, 1977. Lecture Notes in Mathematics, Vol. 580.

[11] E. Chabi and G. Michaille. Random Dirichlet problem: scalar Darcy's law. Potential Anal., 4(2):119-140, 1995.

[12] J. L. Doob. Stochastic processes. Wiley Classics Library. John Wiley \& Sons Inc., New York, 1990. Reprint of the 1953 original, A Wiley-Interscience Publication.

[13] Giovanni P. Galdi. An introduction to the mathematical theory of the Navier-Stokes equations. Vol. I, volume 38 of Springer Tracts in Natural Philosophy. Springer-Verlag, New York, 1994. Linearized steady problems.

[14] David Gérard-Varet. Highly rotating fluids in rough domains. J. Math. Pures Appl. (9), 82(11):1453-1498, 2003.

[15] Willi Jäger and Andro Mikelić. On the roughness-induced effective boundary conditions for an incompressible viscous flow. J. Differential Equations, 170(1):96-122, 2001.

[16] Willi Jäger and Andro Mikelić. Couette flows over a rough boundary and drag reduction. Comm. Math. Phys., 232(3):429-455, 2003.

[17] Willi Jäger, Andro Mikelić, and Nicolas Neuss. Asymptotic analysis of the laminar viscous flow over a porous bed. SIAM J. Sci. Comput., 22(6):2006-2028 (electronic), 2000 .

[18] V. V. Jikov, S. M. Kozlov, and O. A. Oleĭnik. Homogenization of differential operators and integral functionals. Springer-Verlag, Berlin, 1994.

[19] O. A. Ladyženskaja and V. A. Solonnikov. Determination of solutions of boundary value problems for stationary Stokes and Navier-Stokes equations having an unbounded Dirichlet integral. J. Soviet Math., 21:728-761, 1983.

[20] P. Luchini. Asymptotic analysis of laminar boundary-layer flow over finely grooved surfaces. European J. Mech. B Fluids, 14(2):169-195, 1995.

[21] Louis Navier. Sur les lois de l'équilibre et du mouvement des corps élastiques. Mem. Acad. R. Sci. Inst. France, 6:369, 1827. 
[22] George C. Papanicolaou and S. R. S. Varadhan. Diffusions with random coefficients. In Statistics and probability: essays in honor of C. R. Rao, pages 547-552. North-Holland, Amsterdam, 1982.

[23] Kōsaku Yosida. Functional analysis. Classics in Mathematics. Springer-Verlag, Berlin, 1995. Reprint of the sixth (1980) edition. 